\title{
Singlet NMR Methodology in Two-Spin-1/2 Systems
}

\author{
Giuseppe Pileio \\ School of Chemistry, University of Southampton, SO17 1BJ, UK \\ The author dedicates this work to Prof. Malcolm H. Levitt on the occasion of his $60^{\text {th }}$ birthaday
}

\begin{abstract}
This paper discusses methodology developed over the past 12 years in order to access and manipulate singlet order in systems comprising two coupled spin$1 / 2$ nuclei in liquid-state nuclear magnetic resonance. Pulse sequences that are valid for different regimes are discussed, and fully analytical proofs are given using different spin dynamics techniques that include product operator methods, the single transition operator formalism, and average Hamiltonian theory. Methods used to filter singlet order from byproducts of pulse sequences are also listed and discussed analytically. The theoretical maximum amplitudes of the transformations achieved by these techniques are reported, together with the results of numerical simulations performed using custom-built simulation code.
\end{abstract}

Keywords: Singlet States, Singlet Order, Field-Cycling, Singlet-Locking, M2S, SLIC, Singlet Order filtration

\section{Contents}

1 Introduction

2 Nuclear Singlet States and Singlet Order 4

2.1 The spin Hamiltonian and its eigenstates . . . . . . . . . . 4

2.2 Population operators and spin order $\ldots \ldots \ldots \ldots$

2.3 Maximum transformation amplitude . . . . . . . . . . 7

2.4 Spin Hamiltonian in single transition operator formalism . . . . . 8

2.5 Relaxation properties of singlet order . . . . . . . . . . 9

2.5 .1 Coherent dynamics . . . . . . . . . . . . . . . 9

2.5.2 Incoherent dynamics . . . . . . . . . . . . . . . . 9

2.5.3 Central relaxation property of singlet order . . . . . . 10

2.6 Singlet order and magnetic equivalence . . . . . . . . . 11

3 Methods for manipulating singlet order in spin-1/2 pairs $\quad 13$

3.1 Field Cycling . . . . . . . . . . . . . . . . . . . 13

3.1.1 Switching magnetic equivalence by field cycling . . . . . . 13 
3.1.2 Preparation of singlet order . . . . . . . . . . . . . . 14

3.1.3 Conversion of singlet order into observable magnetisation 16

3.2 Singlet-locking . . . . . . . . . . . . . . . . 17

3.2.1 Switching magnetic equivalence by singlet-locking $\ldots . .19$

3.2 .2 Preparation of singlet order . . . . . . . . . . . . 20

3.2.3 Conversion of singlet order into observable magnetisation 22

3.2.4 Preparation and conversion of singlet order by adiabatic switching of the singlet-locking field . . . . . . . . 23

3.3 M2S pulse sequences . . . . . . . . . . . . . 25

3.3.1 J-synchronised spin echoes . . . . . . . . . . . . . . 27

3.3.2 Preparation of singlet order . . . . . . . . . . . 28

3.3.3 Conversion of singlet order into observable magnetisation 30

3.4 SLIC pulse sequences . . . . . . . . . . . . . . . . . 30

3.4.1 Spin-locking induced crossing . . . . . . . . . . . . 31

3.4 .2 Preparation of singlet order . . . . . . . . . . . . 33

3.4.3 Conversion of singlet order into observable magnetisation 33

4 Singlet Order Filtration Techniques 33

$4.1 \quad$ Filtering using polyhedral phase cycling . . . . . . . . . . . 34

$4.2 \quad$ Filtering using field gradient pulses . . . . . . . . . . . . 35

4.3 Filtering using rapid fluctuations in inhomogeneous magnetic fields 37

5 Summary

A Appendices $\quad 39$

A.1 Spherical tensor spin operators . . . . . . . . . . . . . . . 39

A.2 Average Hamiltonian Theory . . . . . . . . . . . . . . . . . 39

A.3 Interaction Frame Transformations . . . . . . . . . . . . 41

A.4 Hermiticity of a combination of Hermitian operators . . . . . . 42

A.5 Single-transition spin operators . . . . . . . . . . . . . 42

A.6 Rotation superoperator simplification for J-synchronised echo trains 43

\section{Introduction}

In a spin- $1 / 2$ pair, singlet order is a form of nuclear spin order that is invariant under rotation and therefore proportional to a zeroth-rank spherical tensor spin operator $[1,2,3]$. This statement carries a series of implications that make singlet order a very interesting form of order in nuclear magnetic resonance spectroscopy. The most important of these implications is that singlet order is long-lived in the sense that once imposed, this order (and the associated polarisation level) can be maintained for a time longer than is possible with longitudinal order, the quantity exploited in conventional NMR and MRI spectroscopy. In technical terms this means that the decay constant of singlet order is often much longer than the decay constant of longitudinal order, known as $\mathrm{T}_{1}[2,3]$. Singlet order is also NMR silent. If access to singlet order is granted, and switching between silent singlet order and observable longitudinal 
order can be done at will, then singlet order may be used as a smart probe, i.e., a probe that can be accessed on demand. These two facts suggest a number of interesting uses of singlet order in NMR and MRI spectroscopy. A number of researchers have been indeed exploiting its long lifetime for the purposeof storage of hyperpolarisation $[4,5,6,7,8,9,10,11,12]$. Hyperpolarisation storage, combined with on-demand accessibility, suggests the use of singlet order as a repository of hyperpolarisation from where the signal enhancement gained by a given hyperpolarisation technique can be repeatedly accessed over a long time (tens of minutes) [12]. The long lifetime of singlet order has also been used to study slow motions like diffusion $[13,14,15,16,17]$, flow [17], molecular dynamics [18, 19] and ligand binding [20, 21, 22, 23].

Since its discovery in the Levitt group $[24,25,26]$ a number of research groups have investigated the field of singlet NMR over the past 12 years with the purpose of defining and characterising singlet order relaxation properties [3, $27,28,29,30]$, and the conditions for its existence [31, 32, 33, 34], and of developing the methodology to access singlet order in different magnetic regimes [24, $26,35,36,37,38,39,9,40,11,41,42,43]$ and to demonstrate the existence of long-lived singlet order in multiple-spin systems [44, 45, 46, 47, 48, 49] and in heteronuclear systems [50]. Significant studies on the chemical requirements for obtaining long-lived spin order have been also conducted [17, 51, 52, 53, 54] with a record lifetime of $70 \mathrm{~min}$ for a ${ }^{13} \mathrm{C}$-pair [54], 26 min for a ${ }^{15} \mathrm{~N}$-pair [51], and $10 \mathrm{~min}$ for a ${ }^{1} \mathrm{H}$-pair [17] demonstrated. The potential use of singlet order in MRI has been discussed and demonstrated [55, 12, 56, 43, 17, 57].

This paper focuses on the efforts of the author and other groups spent in devising methods to access and manipulate singlet order in systems made by two spins-1/2. The aim of the paper is to describe all those techniques and to use spin dynamics concepts, tools and arguments to show how these methods work. The paper is organised as follows. i) Singlet states and singlet order are introduced, their main (relaxation) property is discussed, and the regimes where singlet order is (or not) an eigenstate are defined. ii) Methods developed in order to access and manipulate singlet order are listed and discussed. In each case the propagator for the transformation is explicitly derived by using an appropriate spin dynamics technique. Singlet order manipulation methods include field-cycling, singlet-locking, M2S and SLIC pulse sequences. The adiabatic transformation accomplished by slowly switching on and off a radiofrequency field is also discussed. iii) The three main techniques used in the field to select singlet order from pulse sequence byproducts are discussed. iv) Appendices discusses the main concepts behind various theoretical techniques used in the paper, including single-transition spin operators and average Hamiltonian theory. 


\section{Nuclear Singlet States and Singlet Order}

\subsection{The spin Hamiltonian and its eigenstates}

The spin Hamiltonian for a pair of spins- $1 / 2$ in the liquid state can be written in the rotating frame as:

$$
H=\frac{1}{2} \omega_{\Sigma}\left(\mathbf{I}_{1 z}+\mathbf{I}_{2 z}\right)+\frac{1}{2} \omega_{\Delta}\left(\mathbf{I}_{1 z}-\mathbf{I}_{2 z}\right)+\omega_{J} \mathbf{I}_{1} \cdot \mathbf{I}_{2}
$$

with

$$
\begin{aligned}
& \omega_{\Sigma}=\omega_{1}+\omega_{2} \\
& \omega_{\Delta}=\omega_{1}-\omega_{2} \\
& \omega_{J}=2 \pi J_{12}
\end{aligned}
$$

and where $\omega_{j}$ is the angular frequency of the $j-t h$ site and $J_{12}$ the spinspin coupling between the spins in the pair. Because the Hamiltonian above is written in the rotating frame we also have:

$$
\omega_{j}=\omega_{0}\left(1+\delta_{j}\right)-\omega_{r f}
$$

with $\omega_{0}=-\gamma_{j} B_{0}$ being the Larmor frequency, $\delta_{j}$ the isotropic part of the chemical shift tensor, $\omega_{r f}$ the radiofrequency carrier frequency, $\gamma_{j}$ the magnetogyric ratio for spin- $j$ and $B_{0}$ the value of the static magnetic field.

Singlet states are eigenfunctions of the Hamiltonian in Eq. 1 when the size of the scalar coupling $\omega_{J}$ is much bigger than the difference in chemical shift frequency between the two spins, $\omega_{\Delta}$. This is easily proved by diagonalising the matrix representation of the spin Hamiltonian in the basis obtained as simple product of the Zeeman spin functions for a single isolated spin in a magnetic field:

$$
\mathbf{B}_{Z}=\{|\alpha \alpha\rangle,|\beta \alpha\rangle,|\alpha \beta\rangle,|\beta \beta\rangle\}
$$

The matrix representation of the Hamiltonian in Eq. 1 in the Zeeman basis is:

$$
[H]_{\mathbf{B}_{Z}}=\left(\begin{array}{cccc}
\frac{1}{2} \omega_{\Sigma}+\frac{1}{4} \omega_{\Delta} \tan (\theta) & 0 & 0 & 0 \\
0 & -\frac{1}{2} \omega_{\Delta}-\frac{1}{4} \omega_{\Delta} \tan (\theta) & \frac{1}{2} \omega_{\Delta} \tan (\theta) & 0 \\
0 & \frac{1}{2} \omega_{\Delta} \tan (\theta) & \frac{1}{2} \omega_{\Sigma}-\frac{1}{4} \omega_{\Delta} \tan (\theta) & 0 \\
0 & 0 & 0 & -\frac{1}{2} \omega_{\Sigma}+\frac{1}{4} \omega_{\Delta} \tan (\theta)
\end{array}\right)
$$

where the quantity:

$$
\tan (\theta)=\frac{\omega_{J}}{\omega_{\Delta}}
$$


has been introduced for convenience. Upon diagonalization the following energies and eigenstates are obtained:

$$
\begin{array}{ll}
E_{1}=\frac{1}{2}\left(\omega_{\Sigma}+\frac{\omega_{J}}{2}\right) & \Rightarrow|\alpha \alpha\rangle \\
E_{2}=\frac{1}{2}\left(\sqrt{\omega_{\Delta}^{2}+\omega_{J}^{2}}-\frac{\omega_{J}}{2}\right) & \Rightarrow \cos \left(\frac{\theta}{2}\right)|\alpha \beta\rangle+\sin \left(\frac{\theta}{2}\right)|\beta \alpha\rangle \\
E_{3}=-\frac{1}{2}\left(\sqrt{\omega_{\Delta}^{2}+\omega_{J}^{2}}+\frac{\omega_{J}}{2}\right) & \Rightarrow \sin \left(\frac{\theta}{2}\right)|\alpha \beta\rangle-\cos \left(\frac{\theta}{2}\right)|\beta \alpha\rangle \\
E_{4}=-\frac{1}{2}\left(\omega_{\Sigma}-\frac{\omega_{J}}{2}\right) & \Rightarrow|\beta \beta\rangle
\end{array}
$$

For spin systems where $\omega_{J} \ll \omega_{\Delta}$ (weak-coupling), $\theta \rightarrow 0$ and Eq. 7 reduces to:

$$
\begin{aligned}
E_{1} & =\frac{1}{2} \omega_{\Sigma}+\frac{1}{4} \omega_{J} & & \Rightarrow|\alpha \alpha\rangle \\
E_{2} & =\frac{1}{2} \omega_{\Delta}-\frac{1}{4} \omega_{J} & & \Rightarrow|\alpha \beta\rangle \\
E_{3} & =-\frac{1}{2} \omega_{\Delta}-\frac{1}{4} \omega_{J} & & \Rightarrow|\beta \alpha\rangle \\
E_{4} & =-\frac{1}{2} \omega_{\Sigma}+\frac{1}{4} \omega_{J} & & \Rightarrow|\beta \beta\rangle
\end{aligned}
$$

Conversely, for spin systems where $\omega_{J} \gg \omega_{\Delta}$ (strong-coupling), $\theta \rightarrow \frac{\pi}{2}$ and Eq. 7 reduces to:

$$
\begin{array}{lll}
E_{1}=\frac{1}{2} \omega_{\Sigma}+\frac{\omega_{J}}{4} \omega_{J} & \Rightarrow|\alpha \alpha\rangle \\
E_{2}=\frac{1}{4} \omega_{J} & & \Rightarrow \frac{1}{\sqrt{2}}(|\alpha \beta\rangle+|\beta \alpha\rangle) \\
E_{3}=-\frac{3}{4} \omega_{J} & & \Rightarrow \frac{1}{\sqrt{2}}(|\alpha \beta\rangle-|\beta \alpha\rangle) \\
E_{4}=-\frac{1}{2} \omega_{\Sigma}+\frac{1}{4} \omega_{J} & & \Rightarrow|\beta \beta\rangle
\end{array}
$$

The eigenstates in Eq. 9 can be arranged according to their total angular momentum quantum number $I$ to have:

$$
\begin{aligned}
& \left.\begin{array}{ll}
\left|T_{1}\right\rangle & =|\alpha \alpha\rangle \\
\left|T_{0}\right\rangle & =\frac{1}{\sqrt{2}}(|\alpha \beta\rangle+|\beta \alpha\rangle) \\
\left|T_{-1}\right\rangle & =|\beta \beta\rangle
\end{array}\right\} \Rightarrow I=1 \text { (Triplet States) } \\
& \left|S_{0}\right\rangle=\frac{1}{\sqrt{2}}(|\alpha \beta\rangle-|\beta \alpha\rangle) \Rightarrow I=0 \text { (Singlet State) }
\end{aligned}
$$

where the $T$ and $S$ labels are used to indicate triplet and singlet states, respectively whereas the subscripts $\{0,1,-1\}$ refer to the value of $m=\{I, I-1, \ldots,-I\}$. 


\subsection{Population operators and spin order}

Given a spin state $|\phi\rangle$ the population operator for that state is given by:

$$
P_{\phi}=|\phi\rangle\langle\phi|
$$

Accordingly, the population operator for the singlet state in Eq. 10 is:

$$
\begin{aligned}
P_{S_{0}} & =\left|S_{0}\right\rangle\left\langle S_{0}\right| \\
& =\frac{1}{2}(|\alpha \beta\rangle\langle\alpha \beta|-| \beta \alpha\rangle\langle\alpha \beta|-| \alpha \beta\rangle\langle\beta \alpha|+| \beta \alpha\rangle\langle\beta \alpha|) \\
& =\frac{1}{4} \mathbf{1}-\mathbf{I}_{1} \cdot \mathbf{I}_{2} \\
& =\frac{1}{4} \mathbf{1}+\sqrt{3} \mathbf{T}_{00}^{12}
\end{aligned}
$$

where the last equality shows that the singlet population operator is proportional to the rank-0 spherical tensor operator for a spin pair (a list of spherical tensor spin operators for one and two spins is given in Appendix A.1). Analogously, the population operators for the triplet states expressed in spherical tensor operators are:

$$
\begin{aligned}
P_{T_{0}} & =\frac{1}{4} \mathbf{1}-2 \sqrt{\frac{2}{3}} \mathbf{T}_{20}^{12}-\frac{\sqrt{3}}{3} \mathbf{T}_{00}^{12} \\
P_{T_{ \pm 1}} & =\frac{1}{4} \mathbf{1}+\sqrt{\frac{2}{3}} \mathbf{T}_{20}^{12}-\frac{\sqrt{3}}{3} \mathbf{T}_{00}^{12} \pm \frac{1}{2}\left(\mathbf{T}_{10}^{1}+\mathbf{T}_{10}^{2}\right)
\end{aligned}
$$

The population imbalance between the highest and lowest energy level of a two spin-1/2 system is called longitudinal spin order. Its form, in terms of spherical tensor and product operators, is found from Eq. 13:

$$
\begin{aligned}
\mathbf{L O}=P_{T_{1}}-P_{T_{-1}} & =\mathbf{T}_{10}^{1}+\mathbf{T}_{10}^{2} \\
& =\mathbf{I}_{1 z}+\mathbf{I}_{2 z}
\end{aligned}
$$

Singlet order is defined analogously as the population imbalance between the singlet and triplet manifolds i.e.:

$$
\begin{aligned}
\mathbf{S O}=P_{S_{0}}-\frac{1}{3}\left(P_{T_{1}}+P_{T_{0}}+P_{T_{-1}}\right) & =\frac{4}{3} \sqrt{3} \mathbf{T}_{00}^{12} \\
& =-\frac{2}{3}\left(\mathbf{I}_{1}^{+} \mathbf{I}_{2}^{-}+\mathbf{I}_{1}^{-} \mathbf{I}_{2}^{+}+2 \mathbf{I}_{1 z} \mathbf{I}_{2 z}\right)
\end{aligned}
$$

All techniques described in the following have as a purpose the conversion of longitudinal into singlet order and viceversa.

Note that the norm of $\mathbf{S O}$ is $2 / \sqrt{3}$ and therefore this state does not have the same norm as the other operators in the conventional product operator basis, such as $\mathbf{I}_{1 z}+\mathbf{I}_{2 z}$ or $2 \mathbf{I}_{1 z} \mathbf{I}_{2 z}$, for example, whose norm is $\sqrt{2}$.

A relevant quantity for the discussion below is the order:

$$
\begin{aligned}
\mathbf{p S O}=P_{S_{0}}-P_{T_{0}} & =\frac{4}{3} \sqrt{3} \mathbf{T}_{00}^{12}+\frac{2}{3} \sqrt{6} \mathbf{T}_{20}^{12} \\
& =-\left(\mathbf{I}_{1}^{+} \mathbf{I}_{2}^{-}+\mathbf{I}_{1}^{-} \mathbf{I}_{2}^{+}\right)
\end{aligned}
$$


that is a "pseudo" form of singlet order and is the quantity directly prepared by several of the techniques discussed below. pSO has the advantage of having the same norm as LO. Transformations from pSO to SO must be non-unitary, and are effectively done via spin relaxation processes that are notably non-unitary. The projection of $\mathbf{S O}$ onto $\mathbf{p S O}$ is given by the Liouville bracket:

$$
\frac{(\mathbf{S O} \mid \mathbf{p S O})}{(\mathbf{S O} \mid \mathbf{S O})(\mathbf{p S O} \mid \mathbf{p S O})}=\frac{\operatorname{Tr}\left\{\mathbf{S O}^{\dagger} \mathbf{p S O}\right\}}{\operatorname{Tr}\left\{\mathbf{S O}^{\dagger} \mathbf{S O}\right\} \operatorname{Tr}\left\{\mathbf{p S O}^{\dagger} \mathbf{p S O}\right\}}=\sqrt{\frac{2}{3}}
$$

meaning that by preparing pSO in full one can only achieve a maximum of $\sqrt{\frac{2}{3}} \mathbf{S O}$, assuming that the non-unitary transformation that brings pSO into SO is also achievable in full.

\subsection{Maximum transformation amplitude}

The theoretical maximum efficiency of a pulse sequence is here defined as the theoretical maximum amplitude in the transformation between the operator representing the initial state and the operator defining the final state, assuming unitary transformation. Propagation under radiofrequency pulses and internal spin Hamiltonian is unitary since their associated Hamiltonians are Hermitians. For unitary transformations, the maximum transfer amplitude between two operators is given by the Sørensen equation [58, 59]:

$$
A_{A \rightarrow B}^{\max }=\left|\frac{\operatorname{Tr}\left(B^{\dagger} \hat{U} A\right)}{\operatorname{Tr}\left(B^{\dagger} B\right)}\right|_{\max }=\frac{\Lambda_{B} \Lambda_{A}}{\Lambda_{B} \Lambda_{B}}
$$

where $\Lambda_{A}$ is the ordered list of eigenvalues of the operator $A$.

This approach can be used to calculate the maximum efficiency in transformations from $\mathbf{L O}$ to $\mathbf{p S O}$ using:

$$
\begin{aligned}
\Lambda_{\mathbf{L O}} & =\{1,0,0,-1\} \\
\Lambda_{\mathbf{p S O}} & =\{1,0,0,-1\}
\end{aligned}
$$

to get:

$$
A_{\mathbf{L O} \rightarrow \mathbf{p S O}}^{\max }=A_{\mathbf{p S O} \rightarrow \mathbf{L O}}^{\max }=1
$$

The same equation can be used for the maximum transformation amplitude between $\mathbf{L O}$ and SO, which is of clear interest in this paper. However, as shown above, these two operators have different norms and this may lead to a misleading interpretation of the result of Eq. 18. By choosing to normalise both forms of order to unity the following normalised longitudinal and singlet order operators are obtained:

$$
\begin{aligned}
& \mathbf{L O}^{*}=\frac{1}{\sqrt{2}}\left(\mathbf{I}_{1 z}+\mathbf{I}_{2 z}\right) \\
& \mathbf{S O}^{*}=2 \mathbf{T}_{00}^{12}
\end{aligned}
$$


whose ordered list of eigenvalues is:

$$
\begin{array}{r}
\Lambda_{\mathbf{L} \mathbf{O}^{*}}=\frac{1}{\sqrt{2}}\{1,0,0,-1\} \\
\Lambda_{\mathbf{S O}^{*}}=\frac{1}{2 \sqrt{3}}\{2,-1,-1,-1\}
\end{array}
$$

The maximum transformation from $\mathbf{L} \mathbf{O}^{*}$ and $\mathbf{S O}^{*}$ (and vice versa) is therefore:

$$
A_{\mathbf{L O}^{*} \rightarrow \mathbf{S O}^{*}}^{\max }=A_{\mathbf{S O}^{*} \rightarrow \mathbf{L O}}^{\max }=\sqrt{\frac{2}{3}}
$$

The two main results of this section can be summarised as follow: longitudinal order (LO) can be converted fully into "pseudo" singlet order pSO, however, the transformation from longitudinal order into singlet order (SO) can only be accomplished up to a maximum theoretical efficiency of $\sqrt{2 / 3}$. The transformation train LO $\rightarrow$ SO $\rightarrow$ LO therefore can only be accomplished with a maximum theoretical efficiency of $2 / 3$. Note that these efficiencies are only theoretical and neglect spin relaxation and experimental imperfections.

\subsection{Spin Hamiltonian in single transition operator formalism}

The Hamiltonian in Eq. 1 can be rewritten in terms of single transition spin operators [60] (Appendix A.5) as:

$$
\begin{aligned}
H & =H^{S T}+H^{T T} \\
H^{S T} & =\omega_{\Delta} \mathbf{I}_{x}^{S T}-\omega_{J} \mathbf{I}_{z}^{S T}-\frac{1}{4} \omega_{J} \mathbf{1}^{S T} \\
H^{T T} & =\omega_{\Sigma} \mathbf{I}_{z}^{T T}+\frac{1}{4} \omega_{J} \mathbf{1}^{T T}
\end{aligned}
$$

where the superscript $S T$ indicates the subspace made by $\left|S_{0}\right\rangle$ and $\left|T_{0}\right\rangle$ states and $T T$ that made by $\left|T_{1}\right\rangle$ and $\left|T_{-1}\right\rangle$ states. The first two terms in $H^{S T}$ can be combined into an effective field by introducing:

$$
\begin{aligned}
& \omega_{e}^{S T}=\sqrt{\omega_{\Delta}^{2}+\omega_{J}^{2}} \\
& \theta^{S T}=\arctan \left(-\frac{\omega_{\Delta}}{\omega_{J}}\right)
\end{aligned}
$$

and the full Hamiltonian simplified as:

$$
H=\omega_{e}^{S T} \hat{R}_{y}^{S T}\left(\theta^{S T}\right) \mathbf{I}_{z}^{S T}+\omega_{\Sigma} \mathbf{I}_{z}^{T T}+\frac{1}{4} \omega_{J}\left(\mathbf{1}^{T T}-\mathbf{1}^{S T}\right)
$$

The propagator for the free evolution under this Hamiltonian is readily obtained as:

$$
\begin{aligned}
\hat{U}(t, 0) & =e^{-i H t} \\
& =\hat{R}_{y}^{S T}\left(\theta^{S T}\right) \hat{R}_{z}^{S T}\left(\omega_{e}^{S T} t\right) \hat{R}_{y}^{S T}\left(-\theta^{S T}\right) \hat{R}_{z}^{T T}\left(\omega_{\Sigma} t\right) \hat{\Phi}^{S T}\left(-\frac{\omega_{J}}{4} t\right) \hat{\Phi}^{T T}\left(\frac{\omega_{J}}{4} t\right)
\end{aligned}
$$


with

$$
\hat{\Phi}^{\alpha}(\phi)=e^{-i \phi \mathbf{1}^{\alpha}}
$$

Note that in this formalism we have the identity:

$$
\mathbf{p S O}=2 \mathbf{I}_{z}^{S T}
$$

\subsection{Relaxation properties of singlet order}

The dynamics of a nuclear spin ensemble arew governed by the Liouville equation [60]:

$$
\frac{\partial}{\partial t} \rho(t)=\hat{L}(t) \rho(t)
$$

with $\hat{L}(t)$ representing the Liouvillian superoperator for the system written as:

$$
\hat{L}(t) \rho(t)=-i \hat{H}_{c o h}(t)+\hat{H}_{i n c o h}(t)
$$

where $\hat{H}_{c o h}$ is the commutator superoperator that includes all coherent interactions (same for all members of the ensemble) and $\hat{H}_{\text {incoh }}(t)$ is the double commutator superoperator that includes all incoherent interactions (different for different members of the ensemble) giving rise to nuclear spin relaxation.

\subsubsection{Coherent dynamics}

The nuclear spin Hamiltonian for a two-spin-1/2 system in the liquid state has been given in Eq. 1, where the relevant coherent dynamics is described by a chemical shift term $\left(H_{C S}=H_{\Sigma}+H_{\Delta}\right)$ plus a scalar coupling term $\left(H_{J}\right)$. These terms are written below in terms of spherical tensor operators for convenience:

$$
\begin{aligned}
& H_{\Sigma}=\frac{1}{2} \omega_{\Sigma}\left(\mathbf{T}_{10}^{1}+\mathbf{T}_{10}^{2}\right) \\
& H_{\Delta}=\frac{1}{2} \omega_{\Delta}\left(\mathbf{T}_{10}^{1}-\mathbf{T}_{10}^{2}\right) \\
& H_{J}=\omega_{J} \mathbf{T}_{00}^{12}
\end{aligned}
$$

The associated commutator superoperator is simply:

$$
\hat{H}_{c o h}=[H, \ldots]
$$

\subsubsection{Incoherent dynamics}

The incoherent dynamics are the results of a series of fluctuating interactions whose strength and direction is modulated by molecular motions. In general, the total incoherent Hamiltonian operator $H_{\text {incoh }}(t)$ can be expressed as a sum over many mechanisms:

$$
H_{\text {incoh }}=H_{\text {incoh }}^{A}+H_{\text {incoh }}^{B}+\ldots
$$


and its related superoperator expressed as a sum of terms including all possible cross-correlations between mechanisms, as in:

$$
\hat{H}_{\text {incoh }}=\hat{H}_{\text {incoh }}^{A}+\hat{H}_{\text {incoh }}^{B}+\ldots \hat{H}_{\text {incoh }}^{A \times B}+\ldots
$$

The list of existing mechanisms $(\mathrm{A}, \mathrm{B}, \ldots)$ includes intra-pair dipolar coupling (ipDD), out-of-pair dipolar coupling (opDD), chemical shift anisotropy (CSA) and spin-rotation (SR) among others. For the spin systems considered in this paper and for non-viscous solvents a rough hierarchy of the importance these mechanisms have in regulating the relaxation time of longitudinal order can be written as:

$$
\mathrm{ipDD}>>\mathrm{CSA} \geq \mathrm{opDD} \geq \mathrm{SR} \geq \text { others }
$$

Analytical equations for the Hamiltonians associated with all the terms above are known [61] and the contributions to singlet order relaxation due to several of these interactions has been analytically derived [3, 28, 29]. With regard to the discussion below, it is sufficient to give here only the explicit form of the Hamiltonian describing the main relaxation mechanism (ipDD):

$$
\hat{H}_{\text {incoh }}^{\mathrm{ipDD}}=-\frac{6}{5} b_{j k} \tau_{c} \sum_{m=-2}^{2} \hat{T}_{2 m}^{j k} \hat{T}_{2-m}^{j k}
$$

where $b_{j k}$ is the dipolar constant between the two spins, $\tau_{c}$ the rotational correlation time (fast motion limit [61] assumed), and $\hat{T}_{l m}^{j k}$ the spherical tensor commutator superoperator of rank $l$ and component $m$ for a spin pair.

\subsubsection{Central relaxation property of singlet order}

Eq. 30 states that the dynamics of nuclear spin states are regulated by commutator properties. Therefore a state of the system that commutes with a particular coherent or incoherent Hamiltonian is not affected by the interaction that Hamiltonian represents. Eq. 15, 32 and 37 describe singlet order, the coherent interaction and the main incoherent mechanism in the case of a twospin-1/2 system in non-viscous liquids. The commutation properties of spherical tensors can be then used to derive some interesting features of singlet order. Given the following commutation rules:

$$
\begin{aligned}
& {\left[\mathbf{T}_{00}^{j k}, \mathbf{T}_{1 m}^{j}\right]= \pm \sqrt{\frac{2}{3}} \mathbf{T}_{1 \pm m}^{j k}} \\
& {\left[\mathbf{T}_{00}^{j k}, \mathbf{T}_{2 m}^{j k}\right]=0 \quad \forall m \in\{-2,2\}}
\end{aligned}
$$

the following equalities holds:

$$
\begin{aligned}
{\left[\mathbf{S O}, H_{\Delta}\right] } & \neq 0 \\
{\left[\mathbf{S O}, H_{\Sigma}\right] } & =0 \\
{\left[\mathbf{S O}, H_{J}\right] } & =0 \\
{\left[\mathbf{S O}, H_{\text {inco }}^{\text {ipDD }}\right] } & =0
\end{aligned}
$$


The first equation above states that a difference in chemical shift frequency between the two spins in the pair perturbs the singlet order (as discussed in the next section); second and third equations state that $\mathbf{S O}$ is unaffected by the scalar J-coupling and the sum of chemical shift frequency interactions; and the fourth equation states that singlet order is immune to the intra-pair dipolar coupling relaxation mechanism. Therefore, when ipDD happens to be the main relaxation mechanism for longitudinal order (as is often the case for the kind of systems treated here) then $\mathbf{S O}$ is longer-lived than $\mathbf{L O}$, which is the central relaxation property of singlet order. Longitudinal and singlet order are generally affected in a different ways by all other the relaxation terms in Eq. 35, but their discussion is out of the scope of this paper [3, 28, 29, 30].

To set the language for this paper we simply assume that in the systems treated the longitudinal order builds (and decays) following a first-order kinetics characterised by the time constant $T_{1}$. Analogously, singlet order decays with a first-order kinetics characterised by the time constant $T_{S}$. Because singlet order is immune to ipDD, if this term dominates the decay of longitudinal order then $T_{S}>T_{1}$.

\subsection{Singlet order and magnetic equivalence}

Neglecting relaxation, singlet order is an exact eigenstate of the Liouvillian of the system only in conditions of magnetic equivalence, i.e. when the two spins have the same chemical and magnetic environment. However, in these conditions it is not possible to manipulate singlet order via a radiofrequency pulse sequence, because the singlet remains isolated from the triplet manifold even in the presence of a radiofrequency pulse. This can easily be seen in the

simplest case of an isolated spin pair, by considering the total Hamiltonian of the system in the presence of a radiofrequency pulse with nutation frequency $\omega_{n}$ applied, for simplicity, along the x-axis:

$$
\begin{aligned}
H_{T} & =H+H_{r f} \\
& =\frac{1}{2} \omega_{\Sigma}\left(\mathbf{I}_{1 z}+\mathbf{I}_{2 z}\right)+\frac{1}{2} \omega_{\Delta}\left(\mathbf{I}_{1 z}-\mathbf{I}_{2 z}\right)+\omega_{J} \mathbf{I}_{1} \cdot \mathbf{I}_{2}+\omega_{n}\left(\mathbf{I}_{1 x}+\mathbf{I}_{2 x}\right)
\end{aligned}
$$

written in the basis formed by singlet and triplet states of Eq. 10:

$$
\mathbf{B}_{S T}=\left\{\left|S_{0}\right\rangle,\left|T_{0}\right\rangle,\left|T_{1}\right\rangle,\left|T_{-1}\right\rangle\right\}
$$

where it assumes the form:

$$
\left[H_{T}\right]_{\mathbf{B}_{S T}}=\left(\begin{array}{cccc}
-\frac{3}{4} \omega_{J} & \frac{1}{2} \omega_{\Delta} & 0 & 0 \\
\frac{1}{2} \omega_{\Delta} & \frac{1}{4} \omega_{J} & \frac{1}{\sqrt{2}} \omega_{n} & \frac{1}{\sqrt{2}} \omega_{n} \\
0 & \frac{1}{\sqrt{2}} \omega_{n} & \frac{1}{4} \omega_{J}+\frac{1}{2} \omega_{\Sigma} & 0 \\
0 & \frac{1}{\sqrt{2}} \omega_{n} & 0 & \frac{1}{4} \omega_{J}-\frac{1}{2} \omega_{\Sigma}
\end{array}\right)
$$

Eq. 42 shows that a radiofrequency pulse can only manipulate triplet states, and therefore a difference in chemical shift frequencies between the two spins is 


\begin{tabular}{lllll}
\hline \hline Name & Condition & $\begin{array}{l}\text { Singlet is an } \\
\text { eigenstate? }\end{array}$ & $\begin{array}{l}\text { Equivalence } \\
\text { imposed by }\end{array}$ & $\begin{array}{l}\text { Equivalence } \\
\text { removed by }\end{array}$ \\
\hline weak coupling & $\omega_{J} \ll \omega_{\Delta}$ & no & $\begin{array}{l}\text { field cycling } \\
\text { singlet-locking } \\
\text { chemical reaction }\end{array}$ & no action \\
\hline strong coupling & $\omega_{J} \geq \omega_{\Delta}$ & no & $\begin{array}{l}\text { field cycling } \\
\text { singlet-locking } \\
\text { chemical reaction }\end{array}$ & no action \\
\hline near equivalence & $\omega_{J} \gg \omega_{\Delta}$ & good & no action & no action \\
\hline full equivalence & $\omega_{\Delta}=0$ & exact & no action & chemical reaction \\
\hline \hline
\end{tabular}

Table 1: Classification of a spin system in terms of its magnetic equivalence degree, and summary of available methods used to impose/remove magnetic equivalence.

required in order to be able to manipulate the singlet state through its connection with the $\left|T_{0}\right\rangle$ state.

This dichotomy (singlet order is such only in magnetically equivalent spin pairs, but magnetic inequivalence is needed to prepare such order) is at the heart of this paper, which describes the methods developed over the past 12 years in order to resolve it, with the purpose of manipulating singlet order in different magnetic regimes.

Although a precise categorisation is difficult, roughly speaking there are four important regimes to consider. These are classified on the basis of the degree of magnetic inequivalence of the spin pair, measured by comparing the relative sizes of $\omega_{J}$ and $\omega_{\Delta}$. The situation is summarised in Table 1. In weak and strong coupling regimes, the singlet state is not a good eigenstate of the spin Hamiltonian in Eq. 1 and there is no magnetic equivalence between the spins in the pair. In those cases there is access to a singlet-precursor via combinations of radiofrequency pulses and evolution delays. However, magnetic equivalence must be imposed in order to convert this precursor into singlet order and to exploit its features. This procedure of switching on and off magnetic equivalence has been dubbed symmetry switching [2] and it has been implemented in a number of ways using: i) field cycling [25, 26]; ii) singlet-locking [24] or iii) a chemical reaction $[36,42,62]$. As will be clearer below, field cycling and chemical reaction are most convenient in weak and strong coupling regimes when $\omega_{\Delta}$ is large (larger than a few $\mathrm{kHz}$ to give an indicative number). Singlet-locking can be used as a convenient alternative (no need to cycle field or move sample, nor to adjust conditions for a reaction to happen) only for $\omega_{\Delta}$ of the order of $1-2 \mathrm{kHz}$ or less and depending on hardware.

In the case of near equivalence, the singlet state is a good eigenstate of the Hamiltonian so there is no need for symmetry switching. In those cases, however, the coupling between singlet and triplet state is weak and therefore an ad-hoc methodology is needed to manipulate singlet order. The two methods proposed, magnetisation-to-singlet conversion (M2S) [37, 38] and spin-lock induced crossing (SLIC) [40, 41], are discussed below. 
In the case of full equivalence, the singlet state is an exact eigenstate but some form of symmetry switching is required to break this equivalence and to exploit singlet order. This can only be done via a chemical reaction. Because the aim of this paper is to describe the details of the pulse sequences used to manipulate singlet order, the use of chemical reactions to impose and remove symmetry will not be described any further.

Finally, a certain degree of singlet order can be directly generated directly in any of the above regimes by spin hyperpolarisation [63]. The hyperpolarisation process redistributes populations among spin energy levels to an extent that is far from Boltzmann equilibrium. This process builds up a large amount of longitudinal order by populating one of the outer triplet levels more than the other. At the same time it creates a large imbalance between the populations of the singlet state and the triplet manifold, i.e., a large singlet order. If the amount of longitudinal order (polarisation) prepared by hyperpolarisation is denoted as $p$, then the same process leads to an amount $p^{2} / 3$ of (hyperpolarised) singlet order [63]. This idea has been exploited, for example, as a way to build singlet order under fully equivalent conditions, in conjunction with a chemical reaction for detection $[62,64]$.

\section{Methods for manipulating singlet order in spin-1/2 pairs}

\subsection{Field Cycling}

The field cycling (FC) method $[25,26]$ is the only practical method when $\omega_{\Delta}$ is larger than the available $r f$ amplitude (typically $>1 \mathrm{kHz}$, depending on hardware). It allows manipulation of singlet order by imposing (and removing) magnetic equivalence through the adiabatic change of the spin states and populations occuring when a spin system is exposed to a magnetic field whose strength is slowly varied. As explained below, the method requires the preparation of a state that is converted into singlet order upon field cycling. Because it involves the switching of the magnetic field or the movement of the sample between regions with different magnetic fields, the method is not always practical. In addition, field switching (or sample transport) must happen fast compared to $\mathrm{T}_{1}$ in order to preserve spin order during the transformation. The details of this procedure are summarised below.

\subsubsection{Switching magnetic equivalence by field cycling}

Fig. 1 shows the variation in the form and energy of the four spin states of a homonuclear two spin- $1 / 2$ system (not drawn to scale, and assuming positive values for both the gyromagnetic ratio and the scalar coupling constant) as the field is reduced from high field (HF, $\left.\omega_{J} \ll \omega_{\Delta}\right)$ to low field (LF, $\left.\omega_{J} \geq \omega_{\Delta}\right)$ and down to zero field (ZF, $\left.\omega_{\Delta}=0\right)$. These results show how the population of the state $|\alpha \beta\rangle$ can be transferred to the state $\left|S_{0}\right\rangle$ upon lowering the magnetic field to a value where the condition $\omega_{J} \gg \omega_{\Delta}$ holds. In the same process, the populations of the other three states in high field can be transferred into the three triplet states, which are eigenfunction of the Hamiltonian of Eq. 1 in zero 
HF $\quad$ LF $\quad$ ZF

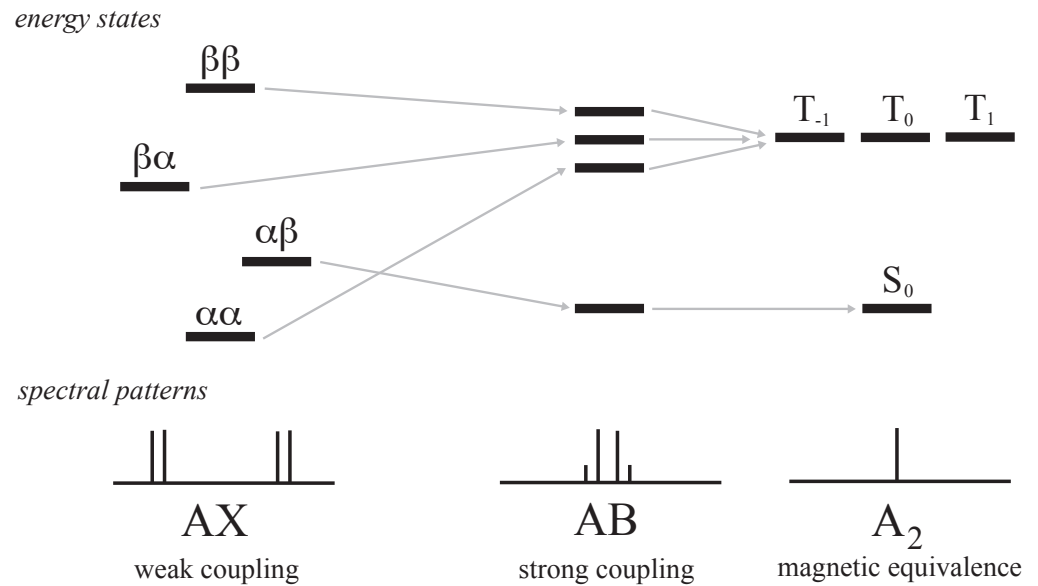

Figure 1: Variation in the energy and form of the four spin states of a homonuclear two spin$1 / 2$ system (not to scale, both gyromagnetic ratio and scalar coupling constant assumed to be positive) as the field is reduce from high field (HF, $\left.\omega_{J} \ll \omega_{\Delta}\right)$ to low field (LF, $\omega_{J} \geq \omega_{\Delta}$ ) to zero field $\left(\mathrm{ZF}, \omega_{\Delta}=0\right)$. The corresponding spectral patterns are shown for convenience.

magnetic field. Accordingly, a population imbalance across the $|\alpha \beta\rangle$ and $|\beta \alpha\rangle$ states in high field results into a population imbalance across $\left|S_{0}\right\rangle$ and $\left|T_{0}\right\rangle$ in low magnetic field, that is pseudo singlet order (pSO). This evolves into singlet order (Eq. 15) as the populations of the three triplet states equilibrate.

The field cycling method can be implemented either by cycling the field between high and low field intensity, or by transporting the sample through regions where the magnetic field has different intensities. In our laboratory, for example, the option to move the sample along the vertical stray field of a conventional superconducting magnet was chosen, for its simplicity and convenience.

\subsubsection{Preparation of singlet order}

Simple inspection of Fig. 1 makes it clear that pSO can be prepared from a population imbalance across the $|\alpha \beta\rangle$ and $|\beta \alpha\rangle$ states. This population imbalance can be prepared via a pulse sequence and a suggested method is reported in Fig. 2. A simple product operator approach [60] can be used to study the evolution of the density operator under each step of the pulse sequence.

Assuming weak coupling (WC) conditions (the regime in which the method is the most useful), the spin Hamiltonian in Eq. 1 can be approximated by:

$$
H \approx H_{W C}=\frac{1}{2} \omega_{\Delta}\left(\mathbf{I}_{1 z}-\mathbf{I}_{2 z}\right)
$$




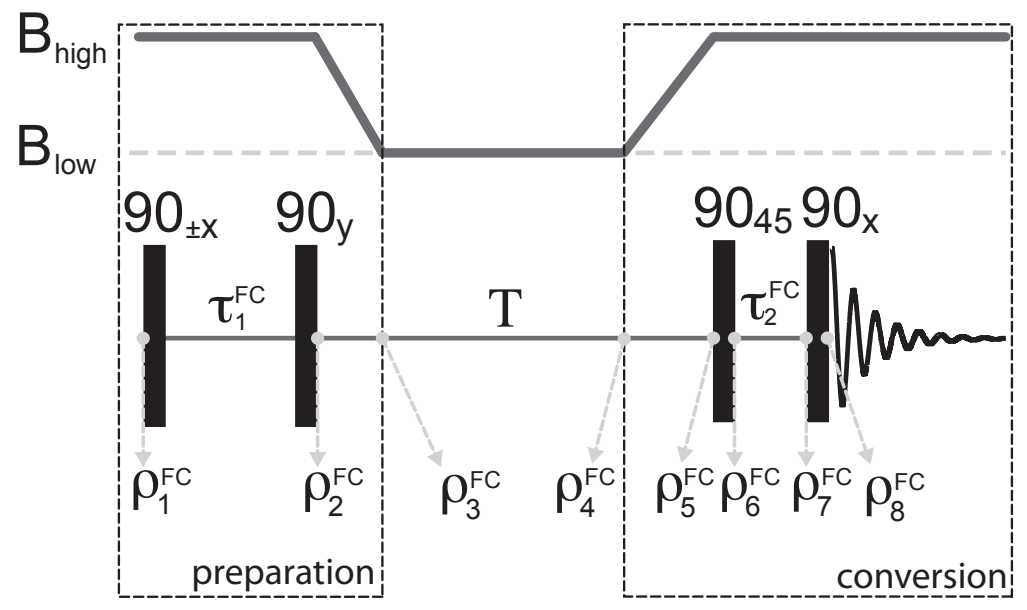

Figure 2: Pulse sequence to manipulate singlet order in weak coupling conditions using a field cycling technique. $\tau_{1}^{F C}=\pi / \omega_{\Delta}$ and $\tau_{2}^{F C}=\pi /\left(2 \omega_{\Delta}\right)$. B Bigh represents a magnetic field intensity where the condition $\omega_{J} \ll \omega_{\Delta}$ holds. Conversely, $B_{\text {low }}$ represents a magnetic field intensity where the condition $\omega_{J} \gg \omega_{\Delta}$ holds.

where the scalar coupling term has been dropped because of the weak coupling condition $\left(\omega_{J} \ll \omega_{\Delta}\right)$ and where the term proportional to $\omega_{\Sigma}$ has been removed since the rf carrier is assumed to be set in the middle of the spectrum, i.e., for $\omega_{\Sigma}=0$. The propagator under this Hamiltonian is readily obtained:

$$
\begin{aligned}
\hat{U}_{W C}(t, 0) & =e^{-i H_{W C} t} \\
& =e^{-\frac{i \omega_{\Delta}}{2}\left(\mathbf{I}_{1 z}-\mathbf{I}_{2 z}\right) t}
\end{aligned}
$$

If $t$ is chosen to fulfil the condition $t=\tau_{1}^{F C}=\pi / \omega_{\Delta}$ then the above propagator represents a rotation of $+90^{\circ}$ for spin 1 and $-90^{\circ}$ for spin 2 about the z-axis of the rotating frame:

$$
\hat{U}_{W C}\left(\tau_{1}^{F C}, 0\right)=\hat{R}_{1 z}(\pi / 2) \hat{R}_{2 z}(-\pi / 2)
$$

The initial state is the longitudinal order given by Eq. 14:

$$
\rho_{1}^{F C}=\mathbf{I}_{1 z}+\mathbf{I}_{2 z}
$$

and evolves under the sequence of events $90 \mathrm{x}-\tau_{1}^{F C}-90 \mathrm{y}$ (Fig. 2) according to:

$$
\begin{aligned}
\rho_{2}^{F C} & =\hat{R}_{y}(\pi / 2) \hat{R}_{1 z}(\pi / 2) \hat{R}_{2 z}(-\pi / 2) \hat{R}_{x}(\pi / 2) \rho_{1}^{F C} \\
& =\mathbf{I}_{1 z}-\mathbf{I}_{2 z}
\end{aligned}
$$

The overall result is equivalent to the effect of a selective $\pi$-pulse on one of the two spins, which is indeed an alternative method to this preparation sequence. 
The resulting density operator $\rho_{2}^{F C}$ can be expressed in terms of population operators as:

$$
\rho_{2}^{F C}=|\alpha \beta\rangle\langle\alpha \beta|-| \beta \alpha\rangle\langle\beta \alpha|
$$

which is the targeted population imbalance.

The adiabatic field cycling adiabatic transformation that follows converts this imbalance into pSO. This transformation can be represented by the following matrix:

$$
\left[R_{H F \rightarrow L F}\right]_{\mathbf{B}_{Z}}=\left(\begin{array}{cccc}
1 & 0 & 0 & 0 \\
0 & \cos (\vartheta / 2) & -\sin (\vartheta / 2) & 0 \\
0 & \sin (\vartheta / 2) & \cos (\vartheta / 2) & 0 \\
0 & 0 & 0 & 1
\end{array}\right)
$$

with $\vartheta=\arctan \left(\omega_{J} /\left(-\gamma B_{0}\left(\delta_{1}-\delta_{2}\right)\right)\right.$ so that $\vartheta \rightarrow-\pi / 2$ when $B_{0} \rightarrow 0$, and the states change as described in Fig. 1. Evolution during field cycling gives:

$$
\begin{aligned}
\rho_{3}^{F C} & =\left(R_{H F \rightarrow L F}\right) \rho_{2}^{F C}\left(R_{H F \rightarrow L F}\right)^{\dagger} \\
& =\left(\left|S_{0}\right\rangle\left\langle S_{0}|-| T_{0}\right\rangle\left\langle T_{0}\right|\right) \\
& =2 \mathbf{I}_{z}^{S T}
\end{aligned}
$$

which coincides with pSO. This state evolves into singlet order (SO) after a non-unitary dissipative transformation (spin relaxation) during a period $\mathrm{T}$ in low magnetic field (with $T_{1} \ll \mathrm{T}<T_{S}$ ):

$$
\begin{aligned}
\rho_{4}^{F C} & =k\left(\left|S_{0}\right\rangle\left\langle S_{0}\left|-\frac{1}{3} \sum_{m}\right| T_{m}\right\rangle\left\langle T_{m}\right|\right) \\
& =k \mathbf{S O}
\end{aligned}
$$

with

$$
k=e^{-\mathrm{T} / T_{S}}
$$

\subsubsection{Conversion of singlet order into observable magnetisation}

Singlet order is non-magnetic and therefore does not give rise to an NMR signal of its own. It must be therefore converted into some form of observable magnetisation via a conversion pulse sequence. Fig. 2 shows one of the possible ways to do this. The method requires the field to be switched back to its high field value (where $\omega_{\Delta} \gg \omega_{J}$ ). Upon adiabatic field switching, the populations of the low-field spin states are transferred into those of high-field spin states as described in Fig. 1 (reading from right to left). Accordingly, the density operator $\rho_{4}^{F C}$ of Eq. 51 transforms into:

$$
\begin{aligned}
\rho_{5}^{F C} & =k\left(|\alpha \beta\rangle\langle\alpha \beta|-\frac{1}{3}(|\alpha \alpha\rangle\langle\alpha \alpha|+| \beta \alpha\rangle\langle\beta \alpha|+| \beta \beta\rangle\langle\beta \beta|)\right) \\
& =-\frac{2}{3} k\left(2 \mathbf{I}_{1 z} \mathbf{I}_{2 z}-\mathbf{I}_{1 z}+\mathbf{I}_{2 z}\right)
\end{aligned}
$$


The first step in the conversion sequence is a $90^{\circ}$ pulse with $45^{\circ}$ phase shift. This produces:

$$
\begin{aligned}
\rho_{6}^{F C} & =\hat{R}_{z}(\pi / 4) \hat{R}_{x}(\pi / 2) \hat{R}_{z}(-\pi / 4) \rho_{5}^{F C} \\
& =-\frac{2}{3} k\left[\frac{1}{\sqrt{2}}\left(\mathbf{I}_{1 y}-\mathbf{I}_{1 x}+\mathbf{I}_{2 x}-\mathbf{I}_{2 y}\right)+\frac{1}{2}\left(2 \mathbf{I}_{1 x} \mathbf{I}_{2 x}-2 \mathbf{I}_{1 x} \mathbf{I}_{2 y}-2 \mathbf{I}_{1 y} \mathbf{I}_{2 x}+2 \mathbf{I}_{1 y} \mathbf{I}_{2 y}\right)\right]
\end{aligned}
$$

This density operator propagates under the Hamiltonian in Eq. 44 for a time $t=\tau_{2}^{F C}=\pi /\left(2 \omega_{\Delta}\right)$, which corresponds to a rotation about the z-axis of $45^{\circ}$ for spin 1 and $-45^{\circ}$ for spin 2 , giving:

$$
\begin{aligned}
\rho_{7}^{F C} & =\hat{R}_{z}(\pi / 4) \hat{R}_{z}(-\pi / 4) \rho_{6}^{F C} \\
& =\frac{2}{3} k\left(2 \mathbf{I}_{1 x} \mathbf{I}_{2 y}+\mathbf{I}_{1 x}+\mathbf{I}_{2 y}\right)
\end{aligned}
$$

The following $90^{\circ}$ pulse about the $\mathrm{x}$-axis produces:

$$
\rho_{8}^{F C}=\frac{2}{3} k\left(2 \mathbf{I}_{1 x} \mathbf{I}_{2 z}+\mathbf{I}_{1 x}+\mathbf{I}_{2 z}\right)
$$

which corresponds to the sum of an antiphase and an in-phase signal at the chemical shift of spin 1 (first two terms) plus a non-detectable term of spin 2 . By changing the phase of the last pulse by $90^{\circ}$, the opposite can be obtained, with the sum of an antiphase and an in-phase signal at the chemical shift of spin 2 plus a non-detectable term of spin 1.

In addition, the phase of the first pulse in the preparation sequence is cycled between $+\mathrm{x}$ and $-\mathrm{x}$ while the receiver phase is cycled between $+\mathrm{x}$ and $\mathrm{x}$, so as to ensure that any two-spin order contribution that could be generated during as a result of cross-correlated relaxation is cancelled out and does not contribute to the final spectrum. This simple trick is applied to all the methods described in this paper.

Note that the conversion sequence discussed here is slightly different from the one that was originally derived. This is because the original paper intended to show a clean separation of signals derived from singlet order through the term $2 \mathbf{I}_{1 z} \mathbf{I}_{2 z}$, which requires a different sequence of events. Other methods to convert $\rho_{5}^{F C}$ into some form of observable magnetisation include the use of a small flip angle pulse or the use of a selective $90^{\circ}$ pulse. Fig. 3 shows a numerical simulation of the full sequence in Fig. 2 where the trajectories of $\mathbf{I}_{1 z}-\mathbf{I}_{2 z}, \mathbf{S O}, 2 \mathbf{I}_{1 x} \mathbf{I}_{2 z}$ and $\mathbf{I}_{1 x}$ are plotted as a function of time for $\omega_{J} /(2 \pi)=$ $50 \mathrm{~Hz}, \omega_{\Delta} /(2 \pi)=5 \mathrm{kHz}, \omega_{\Sigma}=0, \mathrm{~B}_{\text {low }}=0$.

\subsection{Singlet-locking}

An alternative to field cycling consists in imposing magnetic equivalence by applying a radiofrequency field (constant or modulated) [24]. The effect of such a field is to suppress to first order (in the sense of average Hamiltonian theory) the chemical shift terms in the Hamiltonian of Eq. 1. Because this radiofrequency field has the effect of locking the singlet state so as to prevent its mixing with 
a
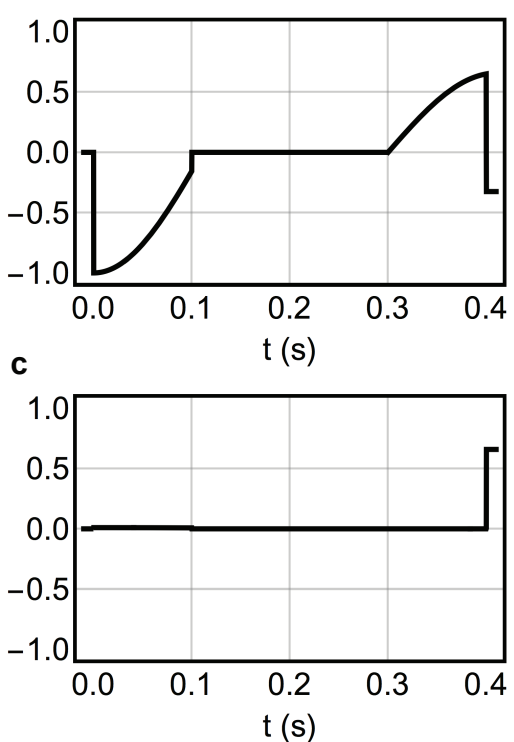

b
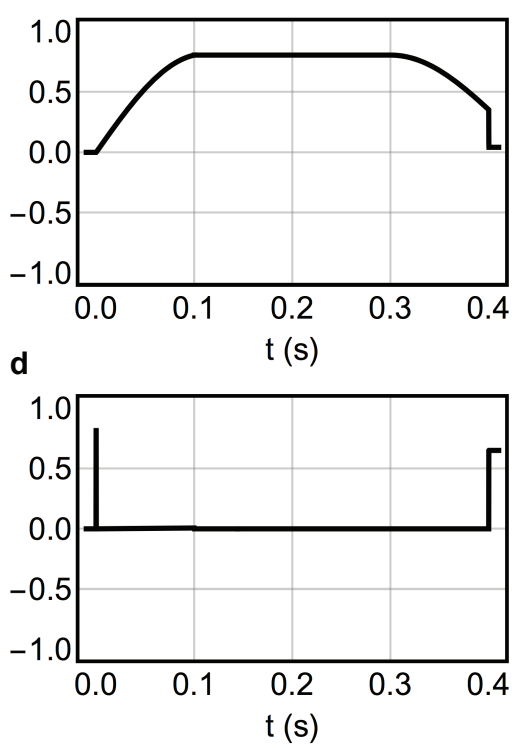

Figure 3: A numerical simulation (custom code, uses SpinDynamica routines [65]) of the evolution of $\mathbf{I}_{1 z}-\mathbf{I}_{2 z}$ (a), SO (b), $2 \mathbf{I}_{1 x} \mathbf{I}_{2 z}$ (c) and $\mathbf{I}_{1 x}$ (d) during the pulse sequence in Fig. 2 for $\omega_{J} /(2 \pi)=50 \mathrm{~Hz}, \omega_{\Delta} /(2 \pi)=5 \mathrm{kHz}, \omega_{\Sigma}=0, \mathrm{~B}_{\text {low }}=0$ and $\mathrm{T}=200 \mathrm{~ms}$. 
the triplet states (which would otherwise happen if a chemical shift term were present in the Hamiltonian) the method takes the name of singlet-locking (some authors prefer to use the word sustaining instead).

\subsubsection{Switching magnetic equivalence by singlet-locking}

A detailed theoretical justification of how singlet-locking works makes use of average Hamiltonian theory [66] whose main equations are summarised in Appendix A.2. The purpose of the following demonstration is therefore to show that the first-order average Hamiltonian of the system does not contain any chemical shift contributions when an unmodulated radiofrequency field is applied on resonance (in the middle of the spectrum, i.e., for $\omega_{\Sigma}=0$ ).

The total Hamiltonian in the presence of an unmodulated field (applied, for simplicity, along the x-axis of the rotating frame) has been given in Eq. 40. On the assumption that $\omega_{n} \gg \omega_{\Delta}$ (which is required for the technique to work; the subscript $n$ stands for nutation) it is convenient to choose the following interaction-frame Hamiltonian:

$$
H_{I F}=\frac{1}{2} \omega_{\Sigma}\left(\mathbf{I}_{1 z}+\mathbf{I}_{2 z}\right)+\omega_{n}\left(\mathbf{I}_{1 x}+\mathbf{I}_{2 x}\right)
$$

which can be conveniently rewritten as:

$$
H_{I F}=\omega_{e}^{S L} \hat{R}_{y}\left(\theta^{S L}\right)\left(\mathbf{I}_{1 z}+\mathbf{I}_{2 z}\right)
$$

using the following definitions:

$$
\begin{aligned}
\omega_{e}^{S L} & =\sqrt{\omega_{n}^{2}+\left(\omega_{\Sigma} / 2\right)^{2}} \\
\theta^{S L} & =\arctan \left(2 \omega_{n} / \omega_{\Sigma}\right)
\end{aligned}
$$

The associated propagator can be readily written as:

$$
\begin{aligned}
\hat{U}_{I F}(t, 0) & =e^{-i \omega_{e}^{S L} \hat{R}_{y}\left(\theta^{S L}\right)\left(\mathbf{I}_{1 z}+\mathbf{I}_{2 z}\right) t} \\
& =\hat{R}_{y}\left(\theta^{S L}\right) \hat{R}_{z}\left(\omega_{e}^{S L} t\right) \hat{R}_{y}\left(-\theta^{S L}\right)
\end{aligned}
$$

Eq. A.19 of Appendix A.2 gives the recipe to derive the representation of the Hamiltonian of Eq. 40 in the frame defined by the interaction Hamiltonian of Eq. 57, as:

$$
\begin{aligned}
\widetilde{H}_{T} & =\frac{1}{2} \omega_{\Delta} \hat{R}_{y}\left(\theta^{S L}\right) \hat{R}_{z}\left(\omega_{e}^{S L} t\right) \hat{R}_{y}\left(-\theta^{S L}\right)\left(\mathbf{I}_{1 z}-\mathbf{I}_{2 z}\right) \hat{R}_{y}\left(\theta^{S L}\right) \hat{R}_{z}\left(\omega_{e}^{S L} t\right) \hat{R}_{y}\left(-\theta^{S L}\right) \\
& +\omega_{J} \mathbf{I}_{1} \cdot \mathbf{I}_{2}
\end{aligned}
$$

where the scalar term remains unchanged because it commutes with all operators in the interaction frame transformation. The first term consists of three nested rotation sandwiches that can be applied consecutively, from the inner to the 
outer, to give:

$$
\begin{aligned}
\widetilde{H}_{T} & =\frac{1}{2} \omega_{\Delta}\left\{\cos ^{2}\left(\theta^{S L}\right)\left(\mathbf{I}_{1 z}-\mathbf{I}_{2 z}\right)+\sin \left(\theta^{S L}\right) \cos \left(\theta^{S L}\right)\left(\mathbf{I}_{1 x}-\mathbf{I}_{2 x}\right)\right. \\
& -\sin \left(\theta^{S L}\right) \cos \left(\omega_{e}^{S L} t\right)\left[\cos \left(\theta^{S L}\right)\left(\mathbf{I}_{1 x}-\mathbf{I}_{2 x}\right)-\sin \left(\theta^{S L}\right)\left(\mathbf{I}_{1 z}-\mathbf{I}_{2 z}\right)\right] \\
& \left.-\sin \left(\theta^{S L}\right) \sin \left(\omega_{e}^{S L} t\right)\left(\mathbf{I}_{1 y}-\mathbf{I}_{2 y}\right)\right\}+\omega_{J} \mathbf{I}_{1} \cdot \mathbf{I}_{2}
\end{aligned}
$$

The first-order average Hamiltonian is calculated from Eq. A.13 using $t_{c}=\frac{2 \pi}{\omega_{n}}$ as the period of the interaction frame Hamiltonian, giving:

$$
\begin{aligned}
\bar{H}_{T}^{(1)} & =\frac{\omega_{n}}{2 \pi} \int_{0}^{\frac{2 \pi}{\omega_{n}}} \widetilde{H}_{T} d t \\
& =\frac{1}{2} \omega_{\Delta} \cos \left(\theta^{S L}\right) \hat{R}_{y}\left(\theta^{S L}\right)\left(\mathbf{I}_{1 z}-\mathbf{I}_{2 z}\right)+\omega_{J} \mathbf{I}_{1} \cdot \mathbf{I}_{2}
\end{aligned}
$$

Given the definition of $\theta^{S L}$ in Eq. 59, for sufficiently strong radiofrequency fields (i.e. for $\left.\omega_{n} \gg \omega_{\Delta}\right)$ and for on-resonance irradiation $\left(\omega_{\Sigma}=0\right), \theta^{S L}=\pi / 2$ so that the first-order averaged Hamiltonian simplifies to:

$$
\bar{H}_{T}^{(1)}=\omega_{J} \mathbf{I}_{1} \cdot \mathbf{I}_{2}
$$

The effect of off-resonance irradiation and the use of radiofrequency fields which are weaker than $\omega_{\Delta}$ have been reported $[67,27,68]$. These studies find that the strength of the singlet-locking field must be about 5 times greater than $\omega_{\Delta}$ in order to achieve good suppression of the chemical shift term in the Hamiltonian. For this reason the singlet-locking approach is practical only when $\omega_{\Delta}$ is of the order of $1-2 \mathrm{kHz}$ or less, since higher values of $\omega_{\Delta}$ would require fields $\omega_{n}$ that are too big to be applied for periods of seconds to minutes, and could stress the probe and increase the sample temperature. The use of modulated radiofrequency irradiation schemes has also been explored, finding that those are effective in increasing the bandwidth of the spin-locking with respect to the sum of the two offsets but not with respect to their difference [27].

\subsubsection{Preparation of singlet order}

When the singlet-locking procedure is used to impose magnetic equivalence and access singlet order, a suitable pulse sequence must be used to create a singlet order precursor that transforms into $\mathbf{S O}$ once singlet-locking irradiation is turned on and magnetic equivalence is imposed. There are multiple choices for such a precursor, the most convenient one consisting in preparing the term: $\mathbf{I}_{1 x} \mathbf{I}_{2 x}+\mathbf{I}_{1 y} \mathbf{I}_{2 y}-2 \mathbf{I}_{1 z} \mathbf{I}_{2 z}$ [18]. This can be done by using the preparation block in Fig. 4.

The starting density operator coincides with longitudinal magnetisation:

$$
\rho_{1}^{S L}=\mathbf{I}_{1 z}+\mathbf{I}_{2 z}
$$

The first $90^{\circ}$ pulse in Fig. 4 creates transverse magnetisation:

$$
\rho_{2}^{S L}=-\left(\mathbf{I}_{1 y}+\mathbf{I}_{2 y}\right)
$$




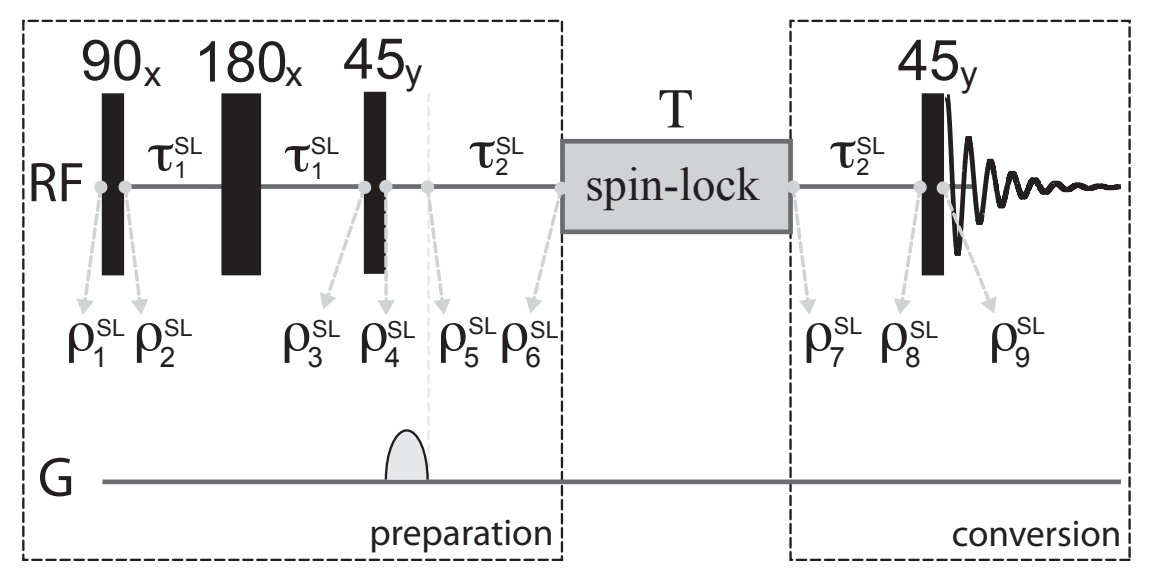

Figure 4: Pulse sequence to manipulate singlet order for weakcoupling conditions using a singlet-locking technique. $\tau_{1}^{S L}=\pi /\left(2 \omega_{J}\right)$ and $\tau_{2}^{S L}=\pi / \omega_{\Delta}$.

A spin-echo (SE) block with $\tau_{1}^{S L}=\frac{\pi}{2 \omega_{J}}$ follows. Because of weak coupling $\left(\omega_{\Delta} \geq \omega_{J}\right)$ and the long duration $\left(\omega_{\Delta} \tau_{1}^{S L} \gg 1\right)$ (this is the regime for which the sequence is the most useful) the propagator during the SE block can be approximated as [69]:

$$
\hat{U}_{S E}(t, 0) \approx e^{-i\left(\frac{\omega_{J}}{2} 2 \mathbf{I}_{1 z} \mathbf{I}_{2 z} t\right)} \hat{R}_{x}(\pi)
$$

and used to find the density operator at time point 3 to be:

$$
\begin{aligned}
\rho_{3}^{S L} & =\hat{U}_{S E}\left(2 \tau_{1}^{S L}, 0\right) \rho_{2}^{S L} \\
& =2 \mathbf{I}_{1 x} \mathbf{I}_{2 z}+2 \mathbf{I}_{1 z} \mathbf{I}_{2 x}
\end{aligned}
$$

The following $45^{\circ}$ pulse, applied about the y-axis, generate a mixture of zeroand double-quantum terms:

$$
\begin{aligned}
\rho_{4}^{S L} & =2 \mathbf{I}_{1 x} \mathbf{I}_{2 x}-2 \mathbf{I}_{1 z} \mathbf{I}_{2 z} \\
& =\frac{1}{2} \mathbf{I}_{1}^{+} \mathbf{I}_{2}^{-}+\frac{1}{2} \mathbf{I}_{1}^{-} \mathbf{I}_{2}^{+}+\frac{1}{2} \mathbf{I}_{1}^{+} \mathbf{I}_{2}^{+}+\frac{1}{2} \mathbf{I}_{1}^{-} \mathbf{I}_{2}^{-}-2 \mathbf{I}_{1 z} \mathbf{I}_{2 z}
\end{aligned}
$$

where the-double quantum terms can be suppressed by a field gradient pulse, leaving:

$$
\begin{aligned}
\rho_{5}^{S L} & =2 \mathbf{I}_{1 x} \mathbf{I}_{2 x}+2 \mathbf{I}_{1 y} \mathbf{I}_{2 y}-2 \mathbf{I}_{1 z} \mathbf{I}_{2 z} \\
& =\frac{1}{2} \mathbf{I}_{1}^{+} \mathbf{I}_{2}^{-}+\frac{1}{2} \mathbf{I}_{1}^{-} \mathbf{I}_{2}^{+}-2 \mathbf{I}_{1 z} \mathbf{I}_{2 z}
\end{aligned}
$$


This state is left to evolve under the Hamiltonian in Eq. 1 for a time $t=\tau_{2}^{S L}=$ $\frac{\pi}{\omega_{\Delta}}$ giving:

$$
\begin{aligned}
\rho_{6}^{S L} & =-2 \mathbf{I}_{1 x} \mathbf{I}_{2 x}-2 \mathbf{I}_{1 y} \mathbf{I}_{2 y}-2 \mathbf{I}_{1 z} \mathbf{I}_{2 z} \\
& =-\frac{1}{2} \mathbf{I}_{1}^{+} \mathbf{I}_{2}^{-}-\frac{1}{2} \mathbf{I}_{1}^{-} \mathbf{I}_{2}^{+}-2 \mathbf{I}_{1 z} \mathbf{I}_{2 z}
\end{aligned}
$$

which is the state required.

The advantages of this preparation method over the original method [24] is that it is broadband with respect to the position of the carrier frequency (the original one requires the carrier to be positioned in the middle of the spectrum, $\omega_{\Sigma}=0$ ) allowing, for example, the possibility of studying molecules with multiple sites undergoing dynamic effects, which may have different chemical shifts for different conformations [18].

\subsubsection{Conversion of singlet order into observable magnetisation}

By analogy with Eq. 51, the density operator $\rho_{6}^{S L}$ after a time period $T_{S}<$ $\mathrm{T}<T_{1}$ under spin-locking irradiation becomes:

$$
\begin{aligned}
\rho_{7}^{S L} & =k\left(\left|S_{0}\right\rangle\left\langle S_{0}\left|-\frac{1}{3} \sum_{m}\right| T_{m}\right\rangle\left\langle T_{m}\right|\right) \\
& =-\frac{2}{3} k\left(2 \mathbf{I}_{1 x} \mathbf{I}_{2 x}+2 \mathbf{I}_{1 y} \mathbf{I}_{2 y}+2 \mathbf{I}_{1 z} \mathbf{I}_{2 z}\right)
\end{aligned}
$$

This state can be converted into antiphase signals at the two chemical shifts by the simple conversion sequence in Fig. 4. The first event in this sequence is free evolution under the Hamiltonian in Eq. 1 for a time $t=\tau_{2}^{S L}=\pi /\left(\omega_{\Delta}\right)$. As described above, this free evolution period changes the sign of the zero-quantum part of the signal, giving:

$$
\rho_{8}^{S L}=-\frac{2}{3} k\left(-2 \mathbf{I}_{1 x} \mathbf{I}_{2 x}-2 \mathbf{I}_{1 y} \mathbf{I}_{2 y}+2 \mathbf{I}_{1 z} \mathbf{I}_{2 z}\right)
$$

This state is converted by the following $45^{\circ}$ pulse into:

$$
\rho_{9}^{S L}=-\frac{2}{3} k\left(2 \mathbf{I}_{1 z} \mathbf{I}_{2 x}+2 \mathbf{I}_{1 x} \mathbf{I}_{2 z}-2 \mathbf{I}_{1 y} \mathbf{I}_{2 y}\right)
$$

where the first two terms correspond to antiphase single-quantum signals at the chemical shifts of the two nuclei in the spin pair and the third term represents undetectable double-quantum coherence.

Fig. 5 shows a numerical simulation of the full sequence in Fig. 4 where the trajectories of $\mathbf{S O}$ and $2 \mathbf{I}_{1 x} \mathbf{I}_{2 z}+2 \mathbf{I}_{1 z} \mathbf{I}_{2 x}$ are plotted as a function of time for $\omega_{J} /(2 \pi)=50 \mathrm{~Hz}, \omega_{\Delta} /(2 \pi)=1 \mathrm{kHz}, \omega_{\Sigma}=0, \omega_{\mathrm{n}} /(2 \pi)=5 \mathrm{kHz}$. 
a

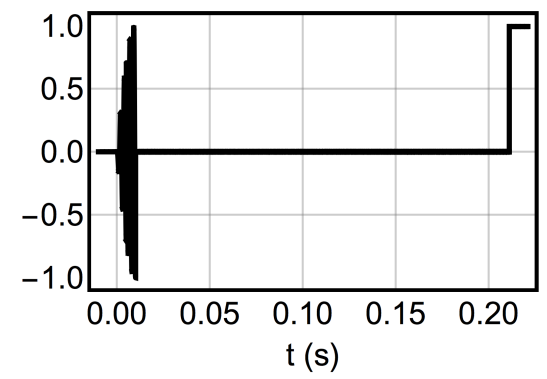

b

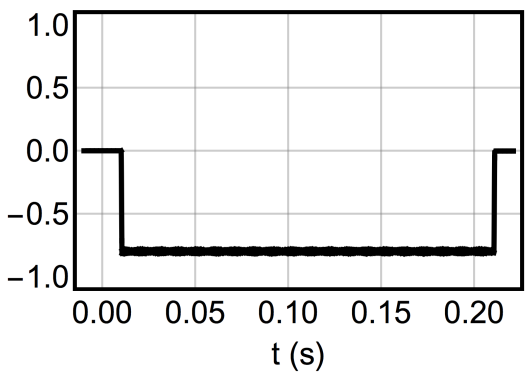

Figure 5: A numerical simulation (custom code, uses SpinDynamica routines [65]) of the evolution of $2 \mathbf{I}_{1 x} \mathbf{I}_{2 z}+2 \mathbf{I}_{1 z} \mathbf{I}_{2 x}$ (a) and SO (b) during the pulse sequence in Fig. 4 for $\omega_{J} /(2 \pi)=$ $50 \mathrm{~Hz}, \omega_{\Delta} /(2 \pi)=1 \mathrm{kHz}, \omega_{\Sigma}=0, \omega_{\mathrm{n}} /(2 \pi)=5 \mathrm{kHz}$ and $\mathrm{T}=200 \mathrm{~ms}$.

\subsubsection{Preparation and conversion of singlet order by adiabatic switching of the} singlet-locking field

An interesting alternative to the preparation (and conversion) sequence described in Section 3.2.2 and 3.2.3 has been introduced by Ivanov et. al. [43, 70, $71]$ and deserves separate attention. The method consists in switching on (for preparation) or off (for conversion) the singlet-locking radiofrequency field in an adiabatic manner, so that the populations of the spin states are transferred without creating any coherences. Such an adiabaticity criterion can be strictly formulated [71] but is beyond the scope of this summary. For our purposes we simply assume that the transformation is adiabatic. Fig. 6 shows the details of the methodology. The conversion from $\mathbf{L O}$ to $\mathbf{p S O}$ is achieved by switching

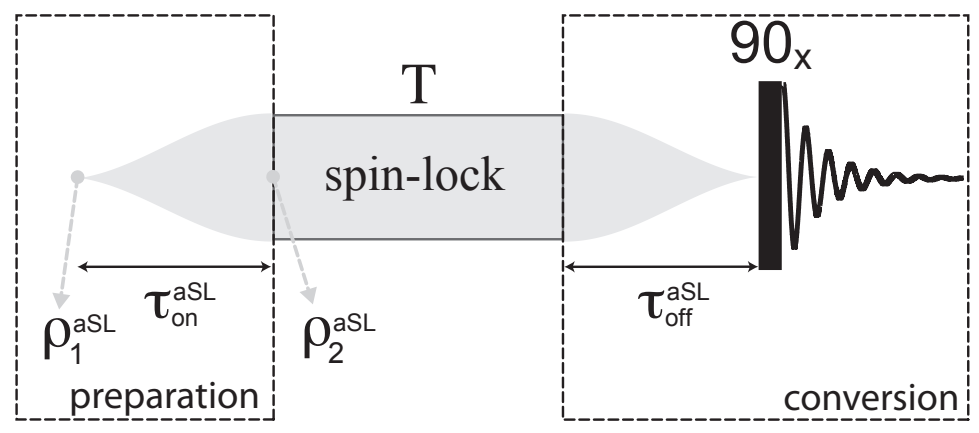

Figure 6:

on a radiofrequency field whose amplitude is increased from zero to $\omega_{n}$ in a time $\tau_{o n}^{a S L}$ chosen to satisfy the adiabaticity criterion. The explicit form for the 
Hamiltonian describing the radiofrequency pulse used in the original paper is:

$$
H_{R F}^{a S L}(t)=\omega_{n}(t)\left(\mathbf{I}_{1 x}+\mathbf{I}_{2 x}\right)+\omega_{o}\left(\mathbf{I}_{1 z}+\mathbf{I}_{2 z}\right)
$$

where the pulse is applied, for simplicity, along the $\mathrm{x}$-axis of the rotating frame at an offset $\omega_{o}$ with:

$$
\omega_{n}(t)=\omega_{n} \sin ^{2}\left(\frac{\pi t}{2 \tau_{o n}^{a S L}}\right)
$$

The total Hamiltonian, including this adiabatically switched pulse, and in the case of weak coupling (the regime where this sequence is the most useful) is:

$$
H^{a S L}=\left(\omega_{1}+\omega_{o}\right) \mathbf{I}_{1 z}+\left(\omega_{2}+\omega_{o}\right) \mathbf{I}_{2 z}+\omega_{J} \mathbf{I}_{1 z} \mathbf{I}_{2 z}-\omega_{n}(t)\left(\mathbf{I}_{1 x}+\mathbf{I}_{2 x}\right)
$$

For $t=0, \omega_{n}(0)=0$, and Eq. 77 assumes the following eigenstates and eigenvalues:

$$
\begin{aligned}
E_{1}(t=0) & =-\frac{1}{2} \omega_{\Delta}-\frac{1}{4} \omega_{J} & & \Rightarrow|\beta \alpha\rangle \\
E_{2}(0) & =\frac{1}{2}\left(\omega_{\Sigma}-2 \omega_{o}\right)+\frac{1}{4} \omega_{J} & & \Rightarrow|\alpha \alpha\rangle \\
E_{3}(0) & =-\frac{1}{2}\left(\omega_{\Sigma}-2 \omega_{o}\right)+\frac{1}{4} \omega_{J} & & \Rightarrow|\beta \beta\rangle \\
E_{4}(0) & =\frac{1}{2} \omega_{\Delta}-\frac{1}{4} \omega_{J} & & \Rightarrow|\alpha \beta\rangle
\end{aligned}
$$

For $t=\tau_{o n}^{a S L}$ one has $\omega_{n}\left(\tau_{o n}^{a S L}\right)=\omega_{n}$; on setting $\omega_{n} \gg \omega_{\Delta}$, the chemical shift term disappears from the total Hamiltonian in a first-oder approximation (see Eq. 64) and the resulting approximated Hamiltonian can be diagonalised to yield the following eigenstates and eigenvalues:

$$
\begin{aligned}
& E_{1}\left(\tau_{o n}^{a S L}\right)=\frac{1}{4} \omega_{J}+\omega_{n} \quad \Rightarrow\left|T_{-1}\right\rangle \\
& E_{2}\left(\tau_{o n}^{a S L}\right)=\frac{1}{4} \omega_{J} \quad \Rightarrow\left|T_{0}\right\rangle \\
& E_{3}\left(\tau_{o n}^{a S L}\right)=-\frac{3}{4} \omega_{J} \quad \Rightarrow\left|S_{0}\right\rangle \\
& E_{4}\left(\tau_{o n}^{a S L}\right)=\frac{1}{4} \omega_{J}-\omega_{n} \quad \Rightarrow\left|T_{1}\right\rangle
\end{aligned}
$$

expressed in a Zeeman basis tilted by $-90^{\circ}$ about the y-axis, where the projection of the total spin along the x-axis is defined. The energies in Eq. 78 and 79 have been arranged from high to low $\left(E_{1}^{0}\right.$ to $\left.E_{4}^{0}\right)$, and this order is maintained provided $\omega_{n}>\omega_{J}$ and $\frac{1}{2}\left(\omega_{\Sigma}+\omega_{\Delta}-\omega_{J}\right)<\omega_{o}<\frac{1}{2} \omega_{\Sigma}$. The order of the states $E_{2}(0)$ and $E_{3}(0)$ is swapped if $\omega_{n}>\omega_{J}$ and $\frac{1}{2}\left(\omega_{\Sigma}-\omega_{\Delta}+\omega_{J}\right)>\omega_{o}>\frac{1}{2} \omega_{\Sigma}$. This means that, provided the offset of the adiabatically switched field remains within the range $\frac{1}{2}\left(\omega_{\Sigma}+\omega_{\Delta}-\omega_{J}\right)<\omega_{o}<\frac{1}{2} \omega_{\Sigma}$, the population of $|\alpha \alpha\rangle$ is transferred into $\left|T_{0}\right\rangle$ and the population of $|\beta \beta\rangle$ is transferred into $\left|S_{0}\right\rangle$ upon varying 
the amplitude of the $r f$ field from 0 to $\omega_{n}$ as described by Eq. 76. On the other hand, in the range $\frac{1}{2}\left(\omega_{\Sigma}-\omega_{\Delta}+\omega_{J}\right)>\omega_{o}>\frac{1}{2} \omega_{\Sigma}$ the population of $|\alpha \alpha\rangle$ is transferred into $\left|S_{0}\right\rangle$ and the population of $|\beta \beta\rangle$ is transferred into $\left|T_{0}\right\rangle$, instead. A population imbalance between $|\alpha \alpha\rangle$ and $|\beta \beta\rangle(\mathbf{L O})$ is therefore transferred into a population imbalance between $\left|S_{0}\right\rangle$ and $\left|T_{0}\right\rangle$ (pSO) upon adiabatic switching of the radiofrequecy field if $\omega_{o}$ lies in one of the intervals indicated above. Note that there is no transfer from $\mathbf{L O}$ to $\mathbf{p S O}$ when $\omega_{o}=\frac{1}{2} \omega_{\Sigma}$, i.e., when the carrier is positioned in the center of the spectrum.

The time dependence of the Hamiltonian in Eq. 77 is such that both its eigenvalues and its eigenstates are time-dependent. This case is dubbed "homogeneous time-dependence", with a propagator given by:

$$
\hat{U}_{a S L}\left(\tau_{o n}^{a S L}, 0\right)=\sum_{r} e^{-i \phi_{r}\left(\tau_{o n}^{a S L}\right)+\mathfrak{b}_{r}}\left|r^{\prime}\right\rangle\langle r|
$$

where:

$$
\phi_{r}\left(\tau_{o n}^{a S L}\right)=\int_{0}^{\tau_{o n}^{a S L}} E_{r}(t) d t
$$

is the dynamic phase and $\mathbb{b}_{r}$ is the geometric phase (also known as Berry's phase), both of which are dependent on the specific shape of the radiofrequency pulse [72]. When $r$ runs through the states in Eq. 78, $r^{\prime}$ runs through the states in Eq. 79, and the initial density operator is comprised of longitudinal order $\left(\rho_{1}^{a S L}=\mathbf{I}_{1 z}+\mathbf{I}_{2 z}\right)$, we have:

$$
\begin{aligned}
\rho_{2}^{a S L} & =\hat{U}_{a S L}\left(\tau_{o n}^{a S L}, 0\right) \rho_{1}^{a S L} \\
& = \begin{cases}-\mathbf{p S O} & \text { for } \frac{1}{2}\left(\omega_{\Sigma}+\omega_{\Delta}-\omega_{J}\right)<\omega_{o}<\frac{1}{2} \omega_{\Sigma} \\
\mathbf{p S O} & \text { for } \frac{1}{2}\left(\omega_{\Sigma}-\omega_{\Delta}+\omega_{J}\right)>\omega_{o}>\frac{1}{2} \omega_{\Sigma}\end{cases}
\end{aligned}
$$

The conversion from singlet order into longitudinal magnetisation is achieved in a similar fashion by adiabatically switching off the radiofrequency field from a value $\omega_{n} \gg \omega_{\Delta}$ to $\omega_{n}=0$ during the time interval $\tau_{\text {off }}^{a S L}$, as shown in Fig. 6 . A suitable shape for the amplitude of the radiofrequency field in this case is:

$$
\omega_{n}(t)=\omega_{n} \cos ^{2}\left(\frac{\pi t}{2 \tau_{o f f}^{a S L}}\right)
$$

and the mechanism of the conversion follows the same arguments as above, here omitted for the sake of brevity. Fig. 7 shows a numerical simulation of the full sequence in Fig. 6 where the trajectories of $\mathbf{L O}$ and $\mathbf{S O}$ have been plotted as a function of time for $\omega_{J} /(2 \pi)=50 \mathrm{~Hz}, \omega_{\Delta} /(2 \pi)=-1 \mathrm{kHz}, \omega_{\mathrm{o}} /(2 \pi)=-100 \mathrm{~Hz}$ and $\omega_{n} /(2 \pi)=5 \mathrm{kHz}$.

\subsection{M2S pulse sequences}

Because singlet states are eigenstates in near-equivalent spin- $1 / 2$ pairs, singlet order is available at high magnetic fields without the need for symmetry 
a

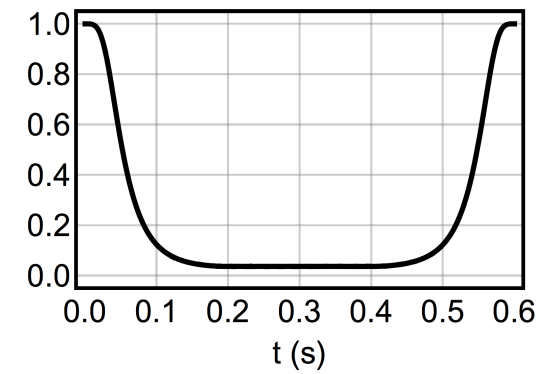

b

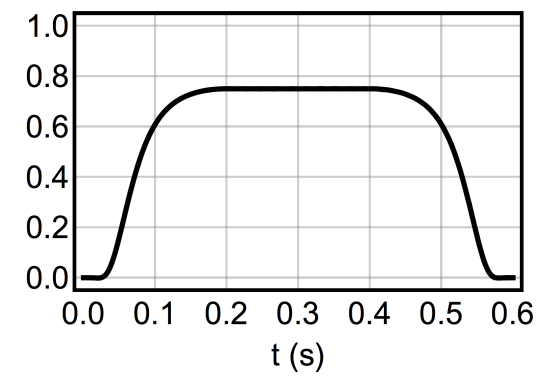

Figure 7: A numerical simulation (custom code, uses SpinDynamica routines [65]) of the evolution of $\mathbf{L O}$ (a) and $\mathbf{S O}$ (b) during the pulse sequence in Fig. 6 for $\omega_{J} /(2 \pi)=50 \mathrm{~Hz}, \omega_{\Delta} /(2 \pi)=$ $-1 \mathrm{kHz}, \omega_{\mathrm{o}} /(2 \pi)=-100 \mathrm{~Hz}, \omega_{\mathrm{n}} /(2 \pi)=5 \mathrm{kHz}, \tau_{\mathrm{on}}^{\mathrm{aSL}}=\tau_{\mathrm{off}}^{\mathrm{aSL}}=200 \mathrm{~ms}$ and $\mathrm{T}=200 \mathrm{~ms}$.

switching under those conditions. Three techniques have been proposed for manipulating singlet order in this regime: (i) magnetisation-to-singlet conversion (M2S) [37, 38] (ii) its reverse, singlet-to-magnetisation (S2M); and (iii) spinlock induced crossing (SLC) [40, 41], which works in both directions. The M2S and S2M pulse sequences can transform longitudinal into singlet order (and vice versa), as shown in Fig. 8. To describe these pulse sequences it is convenient to use the single-transition spin operator formalism described in Appendix A.5, first focusing attention on the effect of the spin-echo block upon which the M2S sequence is built.

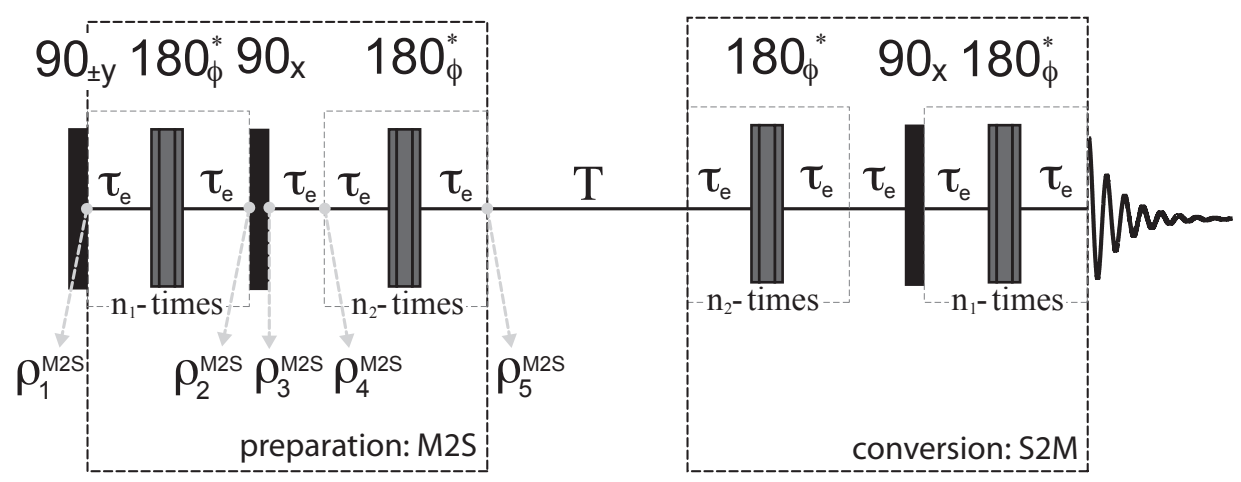

Figure 8: M2S and S2M pulse sequences for manipulating singlet order for nearly-equivalent systems, using J-synchronised spin echoes. 


\subsubsection{J-synchronised spin echoes}

In the nearly-magnetic equivalent regime, $\theta^{S T} \ll 1$ (see Eq. 25), so that free evolution under the propagator in Eq. 27 is an inefficient way to manipulate singlet order. A solution to this problem is provided by J-synchronised spinechoes that, as demonstrated below, provide a more effective way to perform rotations in the singlet-triplet $(S T)$ subspace. M2S and S2M pulse sequences uses J-synchronised spin-echoes to manipulate singlet order in near-equivalence regimes. As in other echo trains, J-synchronised spin-echoes consist of repeated delay/ $\pi$-pulse/delay blocks, but here the delay is synchronised with $\omega_{J}\left(\tau_{e} \approx\right.$ $1 /(4 J))$. In order to write the full propagator under the spin echo, Eq. 27 is used for the free evolution whereas the propagator under a $180^{\circ}$ pulse is best written in the singlet-triplet $(S T)$ and triplet-triplet $(T T)$ subspaces. This can be done by simple inspection of the matrix representation of a $\pi$-pulse in the singlet-triplet basis of Eq. 41:

$$
\left[\hat{R}_{x}(\pi)\right]_{\mathbf{B}_{S T}}=\left(\begin{array}{cccc}
-1 & 0 & 0 & 0 \\
0 & 1 & 0 & 0 \\
0 & 0 & 0 & -1 \\
0 & 0 & -1 & 0
\end{array}\right)
$$

The $\pi$-pulse has therefore the effect of flipping the sign of $\left|T_{0}\right\rangle$, swapping $\left|T_{1}\right\rangle$ and $\left|T_{-1}\right\rangle$ (and flipping their signs) while leaving $\left|S_{0}\right\rangle$ unaltered. In the two $S T$ and $T T$ subspaces this pulse can be then written as a $180^{\circ}$ rotation about the z-axis of the $S T$ subspace followed by a $180^{\circ}$ rotation about the x-axis of the $T T$ subspace, both multiplied by a phase factor:

$$
\hat{R}_{x}(\pi)=\left[\hat{\Phi}^{S T}(-\pi / 2) \hat{R}_{z}^{S T}(\pi)\right]\left[\hat{\Phi}^{T T}(\pi / 2) \hat{R}_{x}^{T T}(\pi)\right]
$$

Eq. 27 and Eq. 85 are now combined together to obtain the propagator under a single spin echo block as:

$$
\begin{aligned}
\hat{U}_{\text {echo }}\left(2 \tau_{e}, 0\right) & =\hat{U}\left(\tau_{e}, 0\right) \hat{R}_{x}(\pi) \hat{U}\left(\tau_{e}, 0\right) \\
& =\hat{U}_{\text {echo }}^{S T}\left(2 \tau_{e}, 0\right) \hat{U}_{\text {echo }}^{T T}\left(2 \tau_{e}, 0\right)
\end{aligned}
$$

with:

$$
\begin{aligned}
\hat{U}_{\text {echo }}^{S T}\left(2 \tau_{e}, 0\right) & =\hat{\Phi}^{S T}\left(-\frac{\pi}{2}-\frac{\omega_{J}}{2} \tau_{e}\right) \hat{R}_{y}^{S T}\left(\theta^{S T}\right) \hat{R}_{z}^{S T}\left(\omega_{e}^{S T} \tau_{e}\right) \hat{R}_{y}^{S T}\left(-\theta^{S T}\right) \\
& \times \hat{R}_{z}^{S T}(\pi) \hat{R}_{y}^{S T}\left(\theta^{S T}\right) \hat{R}_{z}^{S T}\left(\omega_{e}^{S T} \tau_{e}\right) \hat{R}_{y}^{S T}\left(-\theta^{S T}\right) \\
\hat{U}_{\text {echo }}^{T T}\left(2 \tau_{e}, 0\right) & =\hat{\Phi}^{T T}\left(\frac{\pi}{2}+\frac{\omega_{J}}{2} \tau_{e}\right) \hat{R}_{z}^{T T}\left(\omega_{\Sigma} \tau_{e}\right) \hat{R}_{x}^{T T}(\pi) \hat{R}_{z}^{T T}\left(\omega_{\Sigma} \tau_{e}\right)
\end{aligned}
$$

By setting:

$$
\tau_{e}=\frac{\pi}{2 \omega_{e}^{S T}} \approx \frac{\pi}{2 \omega_{J}}
$$


(hence the term J-synchronised) the propagators above simplify to:

$$
\begin{aligned}
\hat{U}_{e c h o}^{S T}\left(2 \tau_{e}, 0\right) & \approx \hat{\Phi}^{S T}\left(-\frac{3 \pi}{4}\right) \hat{R}_{y}^{S T}\left(\theta^{S T}\right) \hat{R}_{z}^{S T}(\pi / 2) \hat{R}_{y}^{S T}\left(-\theta^{S T}\right) \\
& \times \hat{R}_{z}^{S T}(\pi) \hat{R}_{y}^{S T}\left(\theta^{S T}\right) \hat{R}_{z}^{S T}(\pi / 2) \hat{R}_{y}^{S T}\left(-\theta^{S T}\right) \\
\hat{U}_{e c h o}^{T T}\left(2 \tau_{e}, 0\right) & \approx \hat{\Phi}^{T T}\left(\frac{3 \pi}{4}\right) \hat{R}_{z}^{T T}\left(\frac{\omega_{\Sigma} \pi}{2 \omega_{J}}\right) \hat{R}_{x}^{T T}(\pi) \hat{R}_{z}^{T T}\left(\frac{\omega_{\Sigma} \pi}{2 \omega_{J}}\right)
\end{aligned}
$$

For small values of $\theta^{S T}$ (i.e. for near equivalence) the propagator $U_{\text {echo }}^{S T}\left(2 \tau_{e}, 0\right)$ can be approximated by a rotation of $2 \theta^{S T}$ about the x-axis of the $S T$ subspace ( $\hat{R}_{x}^{S T}\left(2 \theta^{S T}\right)$ multiplied by a phase factor (see Appendix A.6 for a full proof). For $\omega_{\Sigma}<<\omega_{J}$ the propagator $U_{e c h o}^{T T}\left(2 \tau_{e}, 0\right)$ can also be approximated by a $\pi$ rotation about the x-axis of the $T T$ subspace, $\hat{R}_{x}^{T T}(\pi)$. The final approximated forms of these propagators are:

$$
\begin{aligned}
& \hat{U}_{\text {echo }}^{S T}\left(2 \tau_{e}, 0\right) \approx \hat{\Phi}^{S T}\left(-\frac{3 \pi}{4}\right) \hat{R}_{x}^{S T}\left(2 \theta^{S T}\right) \\
& \hat{U}_{\text {echo }}^{T T}\left(2 \tau_{e}, 0\right) \approx \hat{\Phi}^{T T}\left(\frac{3 \pi}{4}\right) \hat{R}_{x}^{T T}(\pi)
\end{aligned}
$$

A full rotation of $2 n \theta^{S T}$ radians is achieved when this spin echo block is repeated $n$ times. Therefore the settings:

$$
\begin{aligned}
& n_{1}=\pi /\left(2 \theta^{S T}\right) \\
& n_{2}=\pi /\left(4 \theta^{S T}\right)
\end{aligned}
$$

implement a $180^{\circ}$ and a $90^{\circ}$ rotation about the x-axis of the $S T$ subspace, respectively. A $180^{\circ}$ rotation converts $\left|T_{0}\right\rangle$ into $\left|S_{0}\right\rangle$ and vice versa, whereas a $90^{\circ}$ rotation creates $\left|T_{0}\right\rangle$ and $\left|S_{0}\right\rangle$ coherences. The propagators after $n_{1}$ echoes become:

$$
\begin{aligned}
& {\left[\hat{U}_{\text {echo }}^{S T}\left(2 \tau_{e}, 0\right)\right]^{n_{1}} \approx \hat{\Phi}^{S T}\left(-\frac{3 \pi}{4} n_{1}\right) \hat{R}_{x}^{S T}(\pi)} \\
& {\left[\hat{U}_{\text {echo }}^{T T}\left(2 \tau_{e}, 0\right)\right]^{n_{1}} \approx \hat{\Phi}^{T T}\left(\frac{3 \pi}{4} n_{1}\right) \hat{R}_{x}^{T T}\left(n_{1} \pi\right)}
\end{aligned}
$$

and after $n_{2}$ echoes:

$$
\begin{aligned}
& {\left[\hat{U}_{\text {echo }}^{S T}\left(2 \tau_{e}, 0\right)\right]^{n_{2}} \approx \hat{\Phi}^{S T}\left(-\frac{3 \pi}{4} n_{2}\right) \hat{R}_{x}^{S T}(\pi / 2)} \\
& {\left[\hat{U}_{\text {echo }}^{T T}\left(2 \tau_{e}, 0\right)\right]^{n_{2}} \approx \hat{\Phi}^{T T}\left(\frac{3 \pi}{4} n_{2}\right) \hat{R}_{x}^{T T}\left(n_{2} \pi\right)}
\end{aligned}
$$

\subsubsection{Preparation of singlet order}

J-synchronised spin echo trains are building blocks of M2S pulse sequences that convert transverse magnetisation into pSO (Fig. 8). M2S works on coherence represented by the operator $\mathbf{I}_{1 x}+\mathbf{I}_{2 x}$ and generated from longitudinal 
order by applying of a $90^{\circ}$ radiofrequency pulse about the y-axis. Therefore the starting density operator for the M2S can be expressed as:

$$
\begin{aligned}
\rho_{1}^{M 2 S} & =\mathbf{I}_{1 x}+\mathbf{I}_{2 x} \\
& =\frac{1}{\sqrt{2}}\left[\left(\left|T_{1}\right\rangle+\left|T_{-1}\right\rangle\right)\left\langle T_{0}|+| T_{0}\right\rangle\left(\left\langle T_{1}\right|+\left\langle T_{-1}\right|\right)\right]
\end{aligned}
$$

The first part of the M2S sequence is a J-synchronised echo train with $\tau_{e}=$ $\left(\pi / 2 \omega_{e}^{S T}\right)$ and $n_{1}=\pi /\left(2 \theta^{S T}\right)$, with the propagators in Eq. 92 . Their effect on the triplet states involved in Eq. 94 is:

$$
\begin{gathered}
{\left[\hat{U}_{\text {echo }}^{S T}\left(2 \tau_{e}, 0\right)\right]^{n_{1}}\left|T_{0}\right\rangle=-i e^{i \frac{3 \pi}{4} n_{1}}\left|S_{0}\right\rangle} \\
{\left[\hat{U}_{\text {echo }}^{T T}\left(2 \tau_{e}, 0\right)\right]^{n_{1}}\left(\left|T_{1}\right\rangle+\left|T_{-1}\right\rangle\right)=e^{-i \frac{5 \pi}{4} n_{1}}\left(\left|T_{1}\right\rangle+\left|T_{-1}\right\rangle\right)}
\end{gathered}
$$

The density operator after this first part is:

$$
\begin{aligned}
\rho_{2}^{M 2 S} & =\left[\hat{U}_{\text {echo }}\left(2 \tau_{e}, 0\right)\right]^{n_{1}} \rho_{1}^{M 2 S} \\
& =\frac{i}{\sqrt{2}}\left[\left(\left|T_{1}\right\rangle+\left|T_{-1}\right\rangle\right)\left\langle S_{0}|-| S_{0}\right\rangle\left(\left\langle T_{1}\right|+\left\langle T_{-1}\right|\right)\right]
\end{aligned}
$$

A $90^{\circ}$ pulse is then applied about the $\mathrm{x}$-axis. Its effect on singlet and triplet states is:

$$
\begin{aligned}
\hat{R}_{x}(\pi / 2)\left(\left|T_{1}\right\rangle+\left|T_{-1}\right\rangle\right) & =-i \sqrt{2}\left|T_{0}\right\rangle \\
\hat{R}_{x}(\pi / 2)\left|S_{0}\right\rangle & =\left|S_{0}\right\rangle
\end{aligned}
$$

and the density operator after this transformation is:

$$
\begin{aligned}
\rho_{3}^{M 2 S} & =\hat{R}_{x}(\pi / 2) \rho_{2}^{M 2 S} \\
& =\left(\left|T_{0}\right\rangle\left\langle S_{0}|+| S_{0}\right\rangle\left\langle T_{0}\right|\right) \\
& =2 \mathbf{I}_{x}^{S T}
\end{aligned}
$$

The system is then left to evolve under the internal Hamiltonian for a time $\tau_{e}=$ $\pi /\left(2 \omega_{e}^{S T}\right)$. The effect of free evolution is described in Eq. 27 and approximates to rotation of $-90^{\circ}$ (note the sign of $\theta^{S T}$ in Eq. 25) about the z-axis of the $S T$ subspace, giving:

$$
\begin{aligned}
\rho_{4}^{M 2 S} & =\hat{R}_{z}^{S T}(-\pi / 2) \rho_{3}^{M 2 S} \\
& =-2 \mathbf{I}_{y}^{S T}
\end{aligned}
$$

The following J-synchronised echo train with $\tau_{e}=\pi /\left(2 \omega_{e}^{S T}\right)$ and $n_{2}=\pi /\left(4 \theta^{S T}\right)$ leads to a $90^{\circ}$ rotation about the x-axis of the $S T$ subspace that leads to:

$$
\begin{aligned}
\rho_{5}^{M 2 S} & =\left[\hat{U}_{\text {echo }}^{S T}\left(2 \tau_{e}, 0\right)\right]^{n_{2}} \rho_{4}^{M 2 S} \\
& =-2 \mathbf{I}_{z}^{S T}
\end{aligned}
$$


The term $2 \mathbf{I}_{z}^{S T}$ corresponds to pSO (see Eq. 29) and evolves into SO as the populations of the three triplet states equilibrate by spin relaxation. To calculate the theoretical efficiency of an M2S transformation (neglecting relaxation and pulse imperfections) we use the first equality in Eq. 18 with $A=\mathbf{L O}$ and $B=\mathbf{p S O}$ :

$$
\begin{aligned}
A_{\mathbf{L O} \rightarrow \mathbf{p S O}}^{M 2 S} & =\left|\frac{\operatorname{Tr}\left(\mathbf{p S O}^{\dagger} \hat{U}_{M 2 S} \hat{R}_{y}(\pi / 2) \mathbf{L O}\right)}{\operatorname{Tr}\left(\mathbf{p S O} \mathbf{S O}^{\dagger} \mathbf{p S O}\right)}\right| \\
& =\left|\frac{\operatorname{Tr}\left(\mathbf{p S O}^{\dagger} \mathbf{p S O}\right)}{\operatorname{Tr}\left(\mathbf{p S O}^{\dagger} \mathbf{p S O}\right)}\right|=1
\end{aligned}
$$

where $\hat{R}_{y}(\pi / 2)$ represent the $90 y$ pulse used to convert $\mathbf{L O}$ into $\mathbf{I}_{1 x}+\mathbf{I}_{2 x}$. The result in Eq. 101 shows that M2S accomplishes this transformation fully when spin relaxation and any instrumental imperfections are neglected.

\subsubsection{Conversion of singlet order into observable magnetisation}

Under conditions of near magnetic equivalence, the conversion of singlet order into transverse magnetisation can be done via an S2M sequence consisting of the time-reversal of the M2S sequence discussed in Section 3.3.2. Equivalent arguments (not reported here for the sake of brevity) can be used to demonstrate how the S2M sequence works. Fig. 9 shows a numerical simulation of the full sequence of Fig. 8 where the trajectories of $\mathbf{I}_{1 x}+\mathbf{I}_{2 x}$ (a) and $\mathbf{S O}$ (b) are plotted as a function of time for $\omega_{J}=2 \pi 50, \omega_{\Delta}=2 \pi 5, \omega_{\Sigma}=0$.

a

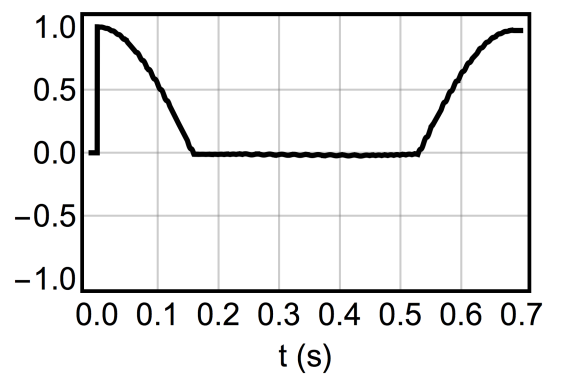

b

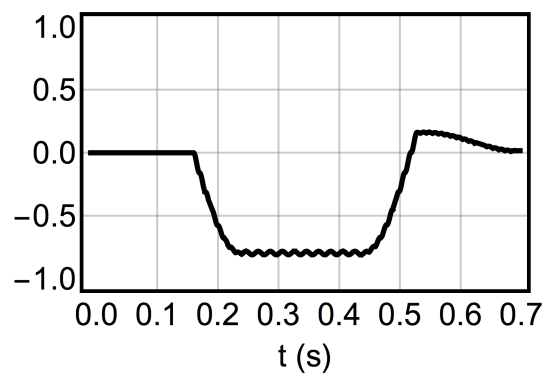

Figure 9: A numerical simulation (custom code, uses SpinDynamica routines [65]) of the evolution of $\mathbf{I}_{1 x}+\mathbf{I}_{2 x}$ (a) and SO (b) during the pulse sequence of Fig. 8 for $\omega_{J} /(2 \pi)=$ $50 \mathrm{~Hz}, \omega_{\Delta} /(2 \pi)=5 \mathrm{~Hz}, \omega_{\Sigma}=0$ and $\mathrm{T}=200 \mathrm{~ms}$. The overshooting of the curve in panel (a) at about $0.5 \mathrm{~s}$ is due to the necessity to round the value of $n_{1}$ to the nearest even integer value.

\subsection{SLIC pulse sequences}

Spin-locking induced crossing (SLIC) $[40,41]$ is an alternative method for manipulating singlet order in near magnetic equivalence regimes. The method 
converts transverse spin order into pSO (and vice versa) and consists in applying a radiofrequency pulse with a nutation frequency that is matched to $\omega_{J}$. This pulse induces oscillations between singlet and triplet states. The pulse length is adjusted so as to maximise the transfer from transverse magnetisation into singlet order. The SLIC pulse sequence is shown in Fig. 10, where the similarity of the preparation and reconversion sequences can be seen.

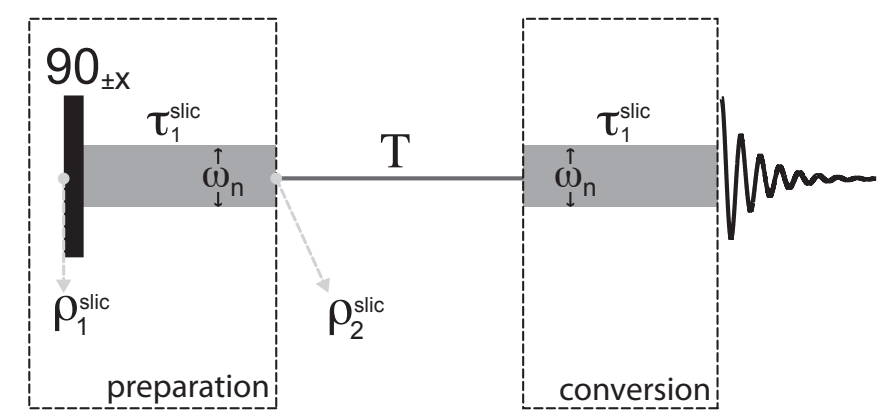

Figure 10: SLIC pulse sequences for manipulating singlet order in near-equivalence conditions. $\tau_{1}^{\text {slic }}=2 \pi /\left(\sqrt{2} \omega_{\Delta}\right)$ and $\omega_{n}=\omega_{J}$.

\subsubsection{Spin-locking induced crossing}

To analyse the effects of the SLIC pulse sequence, we start from the Hamiltonian in Eq. 40 that includes a generic pulse and write its matrix representation in a tilted singlet-triplet basis obtained by rotating the basis in Eq. 41 by $90^{\circ}$ around the y-axis of the laboratory frame. The tilted singlet-triplet basis is:

$$
\begin{aligned}
\mathbf{B}_{S T}^{T} & =\hat{R}_{y}(\pi / 2)\left\{\left|S_{0}\right\rangle,\left|T_{0}\right\rangle,\left|T_{1}\right\rangle,\left|T_{-1}\right\rangle\right\} \\
& =\left\{\left|S_{0}\right\rangle, \frac{1}{\sqrt{2}}\left(\left|T_{1}\right\rangle-\left|T_{-1}\right\rangle\right), \frac{1}{2}\left(\left|T_{1}\right\rangle+\left|T_{-1}\right\rangle\right)-\frac{1}{\sqrt{2}}\left|T_{0}\right\rangle, \frac{1}{2}\left(\left|T_{1}\right\rangle+\left|T_{-1}\right\rangle\right)+\frac{1}{\sqrt{2}}\left|T_{0}\right\rangle\right\}
\end{aligned}
$$

and the Hamiltonian in Eq. 40 takes the form:

$$
\left[H_{T}\right]_{\mathbf{B}_{S T}^{T}}=\left(\begin{array}{cccc}
-\frac{3}{4} \omega_{J} & 0 & -\frac{1}{2 \sqrt{2}} \omega_{\Delta} & \frac{1}{2 \sqrt{2}} \omega_{\Delta} \\
0 & \frac{1}{4} \omega_{J} & 0 & 0 \\
-\frac{1}{2 \sqrt{2}} \omega_{\Delta} & 0 & \frac{1}{4} \omega_{J}-\omega_{n} & 0 \\
\frac{1}{2 \sqrt{2}} \omega_{\Delta} & 0 & 0 & \frac{1}{4} \omega_{J}+\omega_{n}
\end{array}\right)
$$

for the condition $\omega_{\Sigma}=0$, i.e. when the $r f$ carrier is positioned in the center of the spectrum. The tilting of the basis has the effect of placing terms that depend on the nutation frequency of the pulse along the main diagonal. By 
setting $\omega_{n}=\omega_{J}$ the above matrix becomes:

$$
\left[H_{T}\right]_{\mathbf{B}_{S T}^{T}}=\left(\begin{array}{cccc}
-\frac{3}{4} \omega_{J} & 0 & -\frac{1}{2 \sqrt{2}} \omega_{\Delta} & \frac{1}{2 \sqrt{2}} \omega_{\Delta} \\
0 & \frac{1}{4} \omega_{J} & 0 & 0 \\
-\frac{1}{2 \sqrt{2}} \omega_{\Delta} & 0 & -\frac{3}{4} \omega_{J} & 0 \\
\frac{1}{2 \sqrt{2}} \omega_{\Delta} & 0 & 0 & \frac{5}{4} \omega_{J}
\end{array}\right) \text { for }\left(\omega_{\mathrm{n}}=\omega_{J}\right)
$$

The diagonal terms for the first and fourth state in the tilted basis of Eq. 102 are separated by $\left|2 \omega_{J}\right|$ which, in the near magnetic equivalence regime, is much larger than the off-diagonal element that connects these states, $\omega_{\Delta} /(2 \sqrt{2})$. To a first approximation the latter off-diagonal term can be dropped and the Hamiltonian matrix can be approximated as:

$$
\left[H_{T}\right]_{\mathbf{B}_{S T}^{T}} \approx\left(\begin{array}{cccc}
-\frac{3}{4} \omega_{J} & 0 & -\frac{1}{2 \sqrt{2}} \omega_{\Delta} & 0 \\
0 & \frac{1}{4} \omega_{J} & 0 & 0 \\
-\frac{1}{2 \sqrt{2}} \omega_{\Delta} & 0 & -\frac{3}{4} \omega_{J} & 0 \\
0 & 0 & 0 & \frac{5}{4} \omega_{J}
\end{array}\right) \text { for }\left(\omega_{\mathrm{n}}=\omega_{\mathrm{J}}\right)
$$

The sub-matrix that includes the first and third rows (and columns) can now be treated as a two-level system using the single-transition spin operator formalism. In this formalism the Hamiltonian of the system in the presence of a SLIC pulse and in the condition $\omega_{\Sigma}=0$ and $\omega_{n}=\omega_{J}$ is:

$$
H_{T}^{\text {slic }} \approx-\frac{\omega_{\Delta}}{\sqrt{2}} \mathbf{I}_{x}^{13}-\frac{3}{4} \omega_{J} \mathbf{1}^{13}
$$

where the superscript " 13 " refers to the first and third states in Eq. 102 and with the involved singlet transition operators given in Eq. A.23. The associated propagator is readily constructed:

$$
\hat{U}_{\text {slic }}(t, 0)=e^{-i H_{T}^{\text {slic }} t}=\hat{R}_{x}^{13}\left(-\frac{\omega_{\Delta}}{\sqrt{2}} t\right) \hat{\Phi}^{13}\left(-\frac{3}{4} \omega_{J} t\right)
$$

By setting $t=\tau_{1}^{\text {slic }}=2 \pi /\left(\sqrt{2} \omega_{\Delta}\right)$ the propagator takes the form:

$$
\hat{U}_{\text {slic }}\left(\tau_{1}^{\text {slic }}, 0\right)=\hat{R}_{x}^{13}(2 \pi) \hat{\Phi}^{13}(\phi)
$$

with $\phi=-3 \pi \omega_{J} /\left(2 \sqrt{2} \omega_{\Delta}\right)$, representing a rotation of $360^{\circ}$ about the $\mathrm{x}$-axis of the "13" subspace. Its effect on triplet states is:

$$
\begin{aligned}
\hat{U}_{\text {slic }}\left(\tau_{1}^{\text {slic }}, 0\right)\left|T_{0}\right\rangle & =-\frac{i}{\sqrt{2}} e^{i \phi}\left|S_{0}\right\rangle+\frac{1}{2 \sqrt{2}}\left(\left|T_{1}\right\rangle+\left|T_{-1}\right\rangle\right)+\frac{1}{2}\left|T_{0}\right\rangle \\
\hat{U}_{\text {slic }}\left(\tau_{1}^{\text {slic }}, 0\right)\left(\left|T_{1}\right\rangle+\left|T_{-1}\right\rangle\right) & =i e^{i \phi}\left|S_{0}\right\rangle+\frac{1}{2}\left(\left|T_{1}\right\rangle+\left|T_{-1}\right\rangle\right)+\frac{1}{\sqrt{2}}\left|T_{0}\right\rangle
\end{aligned}
$$




\subsubsection{Preparation of singlet order}

The SLIC method works on transverse coherence represented by the operator $\mathbf{I}_{1 x}+\mathbf{I}_{2 x}$ and generated from longitudinal order by the application of a $90^{\circ}$ radiofrequency pulse about the y-axis. Therefore the starting density operator can be expressed as:

$$
\begin{aligned}
\rho_{1}^{\text {slic }} & =\mathbf{I}_{1 x}+\mathbf{I}_{2 x} \\
& =\frac{1}{\sqrt{2}}\left[\left(\left|T_{1}\right\rangle+\left|T_{-1}\right\rangle\right)\left\langle T_{0}|+| T_{0}\right\rangle\left(\left\langle T_{1}\right|+\left\langle T_{-1}\right|\right)\right]
\end{aligned}
$$

This evolves under the propagator of Eq. 108 to give:

$$
\begin{aligned}
\rho_{2}^{\text {slic }} & =\hat{U}_{\text {slic }}\left(\tau_{1}^{\text {slic }}, 0\right) \rho_{1}^{\text {slic }} \\
& =\left[\frac{1}{2}\left(\left|T_{1}\right\rangle+\left|T_{-1}\right\rangle\right)+\frac{1}{\sqrt{2}}\left|T_{0}\right\rangle\right]\left[\frac { 1 } { 2 } \left(\left\langle T_{1}\left|+\left\langle T_{-1}\right|\right)+\frac{1}{\sqrt{2}}\left\langle T_{0}\right|\right]+\left|S_{0}\right\rangle\left\langle S_{0}\right|\right.\right. \\
& =-\frac{4}{3} \sqrt{3} \mathbf{T}_{00}^{12}-\frac{1}{\sqrt{6}} \mathbf{T}_{20}^{12}+\frac{1}{2}\left(\mathbf{T}_{22}^{12}+\mathbf{T}_{2-2}^{12}\right)-\frac{1}{2 \sqrt{2}}\left(\mathbf{T}_{11}^{1}+\mathbf{T}_{1-1}^{1}+\mathbf{T}_{11}^{2}+\mathbf{T}_{1-1}^{2}\right)
\end{aligned}
$$

which contains pSO.

To calculate the theoretical efficiency of a SLIC transformation (neglecting relaxation and pulse imperfections) we use the first equality in Eq. 18 with $A=\mathbf{L O}$ and $B=\mathbf{p S O}$ :

$$
A_{\mathbf{L O} \rightarrow \mathbf{p S O}}^{\text {slic }}=\left|\frac{\operatorname{Tr}\left(\mathbf{S O}^{\dagger} \hat{U}_{\text {slic }}\left(\tau_{1}^{\text {slic }}, 0\right) \hat{R}_{y}(\pi / 2) \mathbf{L O}\right)}{\operatorname{Tr}\left(\mathbf{p S O} \mathbf{S O}^{\dagger} \mathbf{p S O}\right)}\right|=1
$$

where $\hat{R}_{y}(\pi / 2)$ represent the $90 y$ pulse used to convert $\mathbf{L O}$ into $\mathbf{I}_{1 x}+\mathbf{I}_{2 x}$. The result in Eq. 112 shows that neglecting relaxation and imperfections the SLIC method accomplishes the transformation from LO into pSO with the same maximum efficiency as M2S.

\subsubsection{Conversion of singlet order into observable magnetisation}

As shown in Fig. 10, the SLIC pulse sequence can also be used to convert SO back to $\mathbf{I}_{1 x}+\mathbf{I}_{2 x}$, with the same setting as used for preparation of singlet order. The demonstration uses the same argument as in Section 3.4.2 and is skipped here for the sake of brevity. Fig. 11 shows a numerical simulation of the full sequence in Fig. 10 where the trajectories of $\mathbf{I}_{1 x}+\mathbf{I}_{2 x}$ and $\mathbf{S O}$ are plotted as a function of time for $\omega_{J}=2 \pi 50, \omega_{\Delta}=2 \pi 5, \omega_{\Sigma}=0$.

\section{Singlet Order Filtration Techniques}

Methods to prepare singlet order often prepare by-products, as a direct result of the pulse sequence and/or because of instrumental imperfections. In many applications it is convenient to filter out all by-products to retain only singlet order terms. Singlet order filtration can be done in a variety of ways, some of which are discussed below. 

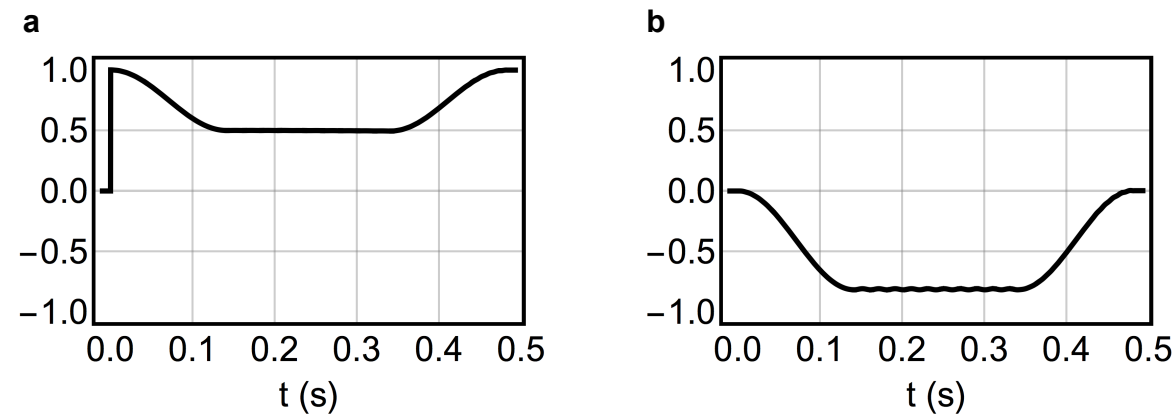

Figure 11: A numerical simulation (custom code, uses SpinDynamica routines [65]) of the evolution of $\mathbf{I}_{1 x}+\mathbf{I}_{2 x}$ (a) and SO (b) during the pulse sequence in Fig. 10 for $\omega_{J} /(2 \pi)=$ $50 \mathrm{~Hz}, \omega_{\Delta} /(2 \pi)=5 \mathrm{~Hz}, \omega_{\Sigma}=0$ and $\mathrm{T}=200 \mathrm{~ms}$.

\subsection{Filtering using polyhedral phase cycling}

Phase cycling is the most common method of signal filtering in NMR. It basically consists in acquiring a number of NMR signals obtained by running many similar pulse sequences which differ only in the phases of one or more radiofrequency pulses. A linear superposition of these signals, multiplied by complex phase factors, can be defined that filters out undesirable signal components while keeping the components with desirable properties. A convenient way to look at these properties uses spherical tensor operators. These operators $\left(\mathbf{T}_{\lambda \mu}^{k}\right.$, see Appendix A.1 for some examples) are described by two quantum numbers, called the rank, denoted $\lambda$, and the component index $\mu$, which takes values $\mu=\{-\lambda,-\lambda+1, \ldots, \lambda\}$. Spin order can be written is terms of such operators where the component index $\mu$ of the irreducible spherical tensor operator corresponds to the coherence order. Traditional phase cycles, which are based on the rotational properties of spin order terms around the z-axis, can only select among terms with different coherence orders (different $\mu$ ) but cannot distinguish among terms with different value of $\lambda$. These methods are therefore not valid for the purpose of selecting singlet order with $\lambda=\mu=0$, as they cannot distinguish singlet order from spin order represented by higher-rank tensors with the same component index $\mu=0$.

The STA (spherical tensor analysis) method [73] instead achieves a complete classification of the spin order terms according to both $\lambda$ and $\mu$, up to a chosen maximum rank. The details of how this method works are described in the original publication [73]; its application for singlet order filtration has been reported [74] and therefore will not be discussed here any further. The general scheme for filtering using polyhedral phase cycles is shown in Fig. 12. It consists of inserting two $90^{\circ}$ radiofrequency pulses with phases $\phi_{1}$ and $\phi_{2}$, and adding an overall phase $\phi_{A}$ to the whole sequence of events that precedes these two pulses and an overall phase $\phi_{B}$ to the whole sequence of events that follows 


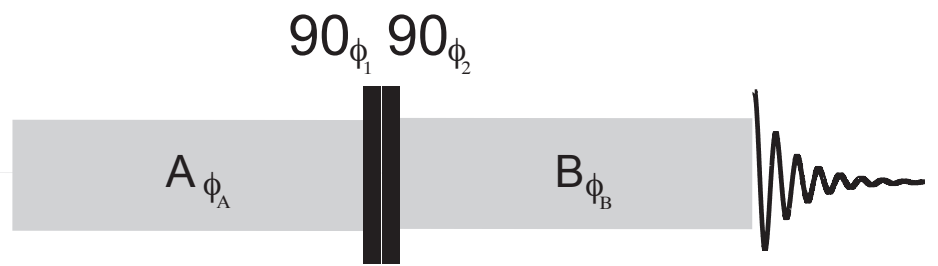

Figure 12: Polyhedral phase filtering implementation scheme done by inserting two $90^{\circ}$ pulses and phase cycling the phases $\phi_{A}$ and $\phi_{B}$ of the two blocks, and the phases $\phi_{1}$ and $\phi_{2}$ of the two inserted pulses according to Table 2 .

these two pulses. Blocks A and B represent, in the context of singlet NMR, the preparation and conversion sequences discussed above, respectively. The phases $\phi_{1}, \phi_{2}, \phi_{A}$ and $\phi_{B}$ are cycled in $\mathcal{N}$ steps such that:

$$
\mathcal{N}=\left(\lambda_{\max }+1\right) \mathcal{V}
$$

where $\lambda_{\max }$ is the highest rank of nuclear spin order with a significant intensity at the junction of sequences $\mathrm{A}$ and $\mathrm{B}$, and $\mathcal{V}$ the number of vertices of a regular polyhedron chosen according to the value of $\lambda_{\max }$, hence the name polyhedral phase cycling.

An ensemble of spin systems each comprising $\mathrm{N}$ coupled spins- $1 / 2$, can only support spin order terms for which both the rank $\lambda$ and the component index $\mu$ are less than or equal to $\mathrm{N}$. This paper deals only with two-spin- $1 / 2$ systems where $\mathrm{N}=2$, so $\lambda_{\max }=N=2$. Under these conditions the polyhedron of choice is the tetrahedron, with $\mathcal{V}=4$ and $\mathcal{N}=12$ [73, 74], meaning that singlet order filtering via polyhedral phase cycling requires a minimum of 12 steps in the phase cycling. The four phases in Fig. 12 are then cycled as shown in Table 2.

The NMR signals for the 12-steps phase cycle are added together with the same weights. This procedure projects out the part of the NMR signal that derives from isotropic spin order terms $(\lambda=\mu=0$, i.e. SO $)$ and that exists at the junction of the two pulse sequence blocks. A drawback of this filtering method is that it is clearly time-consuming and requires multiple transients with reproducible intensities. It could therefore be incompatible with some experiments, such as hyperpolarised NMR/MRI.

\subsection{Filtering using field gradient pulses}

A powerful alternative to phase cycling for signal selection is provided by gradient filtering, which uses field gradient pulses to destroy unwanted spin orders. The advantage of this method for singlet order filtration is that it is a one-shot technique, meaning that it works in a single experiment. For this reason, it may be preferred to the polyhedral phase cycling method above. It does however require gradient hardware for generating gradients in at least one direction. Pulsed field gradients induce rotations around the z-axis of the 


\begin{tabular}{llll}
\hline \hline$\phi_{A}$ & $\phi_{1}$ & $\phi_{2}$ & $\phi_{B}$ \\
\hline 0 & 0 & 180 & 0 \\
120 & 0 & 180 & 0 \\
240 & 0 & 180 & 0 \\
109.47 & 109.47 & 180 & 0 \\
229.47 & 109.47 & 180 & 0 \\
349.47 & 109.47 & 180 & 0 \\
229.47 & 229.47 & 300 & 0 \\
349.47 & 229.47 & 300 & 0 \\
109.47 & 229.47 & 300 & 0 \\
349.47 & 349.47 & 60 & 0 \\
109.47 & 349.47 & 60 & 0 \\
229.47 & 349.47 & 60 & 0 \\
\hline \hline
\end{tabular}

Table 2: Phases $\phi_{A}, \phi_{1}, \phi_{2}$ and $\phi_{B}$ for a 12-step tetrahedral phase cycle, suitable for isotropic filtering of an NMR signal with $\lambda_{\max }=2$. The general pulse sequence scheme in Fig. 12 is used. All angles are given in degrees.

laboratory frame through an angle $\alpha$ that depends on the position in space, according to:

$$
\alpha(\vec{r})=\gamma_{I} \int_{0}^{\tau_{G}} \vec{G}(t) \cdot \vec{r} d t
$$

where $\gamma_{I}$ is the gyromagnetic ratio of the nucleus $I$. If the gradient is sufficiently strong and the spatial distribution of spin states is isotropic, then the z-rotation is uniformly distributed across the sample meaning that all possible values of $\alpha$ are equally sampled. A generic rotation in the $3 \mathrm{D}$ space can be broken down into three successive rotations about the Euler angles $\alpha, \beta$ and $\gamma$ as:

$$
\hat{R}(\alpha, \beta, \gamma)=\hat{R}_{z}(\alpha) \hat{R}_{y}(\beta) \hat{R}_{z}(\gamma)
$$

Such rotations can in effect be implemented by a radiofrequency pulse that leads to a rotation through an angle $\beta$ around the y-axis, preceded and followed by a field gradient pulses, as shown in Fig. 13a.

In this way $\alpha$ and $\gamma$ are uniformly sampled across the sample by the gradient pulses, while the choice of $\beta$ determines the ranks of the tensor components that are filtered out, according to:

$$
d_{00}^{\lambda}(\beta)=0
$$

where $d_{00}^{\lambda}(\beta)$ is the reduced Wigner matrix element of rank $\lambda$ and components $m=0, m^{\prime}=0$, here listed up to rank 2 :

$$
\begin{aligned}
& d_{00}^{0}(\beta)=1 \\
& d_{00}^{1}(\beta)=\cos (\beta) \\
& d_{00}^{2}(\beta)=\frac{1}{2}\left(3 \cos ^{2}(\beta)-1\right)
\end{aligned}
$$


a

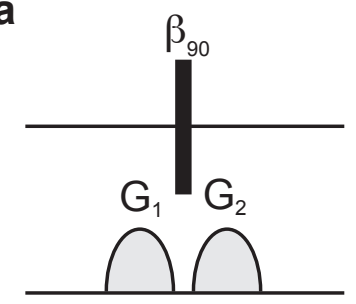

b

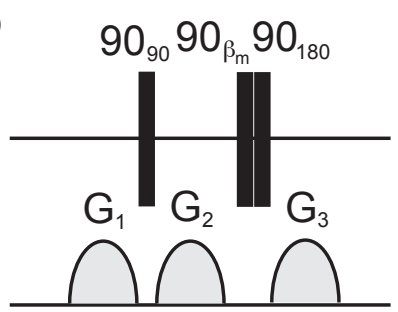

Figure 13: a) Scheme to implement a generic rotation in the 3D spin space using a combination of field gradients and radiofrequency pulses. b) Gradient-based singlet order filtering sequence that eliminates tensors up to rank-3 in two-spin-1/2 systems under near magnetic equivalence conditions. $\beta_{m}=\arctan (\sqrt{2})$ is known as the magic angle.

From Eq. 117 it is immediately clear that the signal component of rank $\lambda=0$ (i.e. singlet order) is always retained. Furthermore, setting $\beta=\pi / 2$ results in filtering out the signal component of rank $\lambda=1$ (and all other odd-rank terms because their reduced Wigner matrix elements are a function of $\cos (\beta)$ ); setting $\beta=\arctan (\sqrt{2})$ results in filtering out the signal component of rank $\lambda=2$, and so on. Such filters can be concatenated to achieve multiple rank filtering, provided that the ranks do not mix during the filtration process.

For the purpose of singlet order filtering in a two-spin-1/2 system, where the maximum achievable rank is 2 , we would therefore combine a filter with $\beta=\pi / 2$ and a filter with $\beta=\arctan (\sqrt{2})$ to effectively eliminate any signal components with $\lambda \neq 0$ and up to $\lambda_{\max }=3$. This filter, originally developed to be used for near-equivalent spin pairs [75], is shown in Fig. 13b, where the pulse with $\beta=\arctan (\sqrt{2})$ has been replaced by an equivalent composite pulse $90_{\beta} 90_{180}$ to obtain a better resolution on the angle. This filter is not suitable for systems with a significant chemical shift difference (large enough that the evolution under the chemical shift Hamiltonian during the second gradient pulse mixes the ranks). In such cases the second gradient may required to be replaced by two gradient pulses of opposite sign sandwiching a refocusing pulse.

\subsection{Filtering using rapid fluctuations in inhomogeneous magnetic fields}

Filtration of singlet order by rapidly fluctuating magnetic fields has been suggested [63] as an alternative to the methods discussed above when filtration has to be accomplished outside of the spectrometer, where radiofrequency pulses and pulse field gradients are not easily accessible/available. This filtering method consists in shaking the sample in the highly inhomogeneous field present inside a mu-metal chamber. Such a chamber is conventionally used to shield from magnetic fields, and is made of an alloy of $\mathrm{Ni}, \mathrm{Fe}$ and other metals typically including $\mathrm{Mo}, \mathrm{Si}$ and $\mathrm{Cu}$ with a very high magnetic permeability $\mu$, hence the name. Materials with such a high magnetic permeability concentrate the magnetic field flux so that the lines of flux are directed through the metal rather than into the enclosed space. This results in an effective shielding of 
the space inside the chamber from any external magnetic fields whose strength is below the mu-metal saturation point (0.76 Tesla for the alloy cited). The field inside the chamber is very low, but is not exactly zero. It can be reduced further by enclosing the mu-metal chamber in another chamber (and repeating this multiple times to obtain better and better shielding properties).

For the purpose of singlet order filtration we are interested in the very small but highly inhomogeneous field that remains within a mu-metal chamber. By moving the sample around such an inhomogeneous and extremely low magnetic field, each voxel of the sample is exposed to a diffrent field history, resulting in a random rotation. On integrating over the whole volume the net result is that any signal component with $\operatorname{rank} \lambda \geq 1$ is completely randomised and integrates to zero, while the rotationally invariant term with $\lambda=0$, which corresponds to singlet order, is left untouched. When compared to the other filtration methods above, this is the most effective way to achieve isotropic filtration, because it effectively destroys any tensor component with rank higher than zero. All other methods have a cut off $\left(\lambda_{\max }\right)$ arising from the need to approximate the complete randomisation by a finite number of phase cycling or gradient filtering steps.

\section{Summary}

In this paper I have been discussing radiofrequency pulse sequences used to access and manipulate singlet-order in two-spin- $1 / 2$ systems that were available at the time of writing. These include: field cycling, spin-locking, adiabatic spinlocking switching, M2S and SLIC. The main techniques used to filter out singlet order from by-products of these pulse sequences are also briefly introduced, including filtering using phase cycling, gradient field pulses and inhomogeneous fields. Full analytical proofs are given in all cases and the conditions in which these sequences are applicable or most conveniently used are stated. The appendices that follow focus on the most relevant features of the spin dynamics techniques invoked in the manuscript. 


\section{A. Appendices}

\section{A.1. Spherical tensor spin operators}

The spherical tensor spin operators for one spin- $1 / 2$ are:

$$
\begin{aligned}
\mathbf{T}_{10}^{j} & =\mathbf{I}_{j z} \\
\mathbf{T}_{1 \pm 1}^{j} & =\mp \frac{1}{\sqrt{2}} \mathbf{I}_{j}^{ \pm}
\end{aligned}
$$

and for a pair of spins- $1 / 2$ :

$$
\begin{aligned}
\mathbf{T}_{00}^{j k} & =-\frac{1}{\sqrt{3}} \mathbf{I}_{j} \mathbf{I}_{k} \\
\mathbf{T}_{10}^{j k} & =\frac{1}{2 \sqrt{2}}\left(\mathbf{I}_{j}^{+} \mathbf{I}_{k}^{-}-\mathbf{I}_{j}^{-} \mathbf{I}_{k}^{+}\right) \\
\mathbf{T}_{1 \pm 1}^{j k} & =-\frac{1}{2}\left(\mathbf{I}_{j}^{ \pm} \mathbf{I}_{k z}-\mathbf{I}_{j z} \mathbf{I}_{k}^{ \pm}\right) \\
\mathbf{T}_{20}^{j k} & =\frac{1}{\sqrt{6}}\left(3 \mathbf{I}_{j z} \mathbf{I}_{k z}-\mathbf{I}_{j} \mathbf{I}_{k}\right) \\
\mathbf{T}_{2 \pm 1}^{j k} & =\mp \frac{1}{2}\left(\mathbf{I}_{j}^{ \pm} \mathbf{I}_{k z}+\mathbf{I}_{j z} \mathbf{I}_{k}^{ \pm}\right) \\
\mathbf{T}_{2 \pm 2}^{j k} & =\frac{1}{2} \mathbf{I}_{j}^{ \pm} \mathbf{I}_{k}^{ \pm}
\end{aligned}
$$

\section{A.2. Average Hamiltonian Theory}

Average Hamiltonian Theory (AHT) provides an approximate solution to the Liouville-von Neumann equation:

$$
\frac{\partial}{\partial t} \rho(t)=-i H(t) \rho(t)
$$

a general solution of which is obtained by recursive integration:

$$
\begin{aligned}
\rho(t) & =\rho(0)+(-i) \int_{0}^{t} H\left(t_{1}\right) \rho\left(t_{1}\right) d t_{1} \\
& =\rho(0)+(-i) \int_{0}^{t}\left(H\left(t_{1}\right) \rho(0)+(-i) \int_{0}^{t} H\left(t_{2}\right) \rho\left(t_{2}\right) d t_{1} d t_{2}\right) \\
& =\ldots
\end{aligned}
$$

The above equation can be compacted as follows:

$$
\begin{aligned}
\rho(t) & =\left[\sum_{n=0}^{\infty} U_{n}(t, 0)\right] \rho(0) \\
\hat{U}_{n}(t, 0) & =(-i)^{n} \int_{0}^{t} d t_{1} \int_{0}^{t_{1}} d t_{2} \ldots \int_{0}^{t_{n-1}} d t_{n} \hat{T} H\left(t_{1}\right) H\left(t_{2}\right) \ldots H\left(t_{n}\right)
\end{aligned}
$$


which is analogous to the Taylor series of an exponential function only if timeordered by the Dyson operator $\hat{T}$ :

$$
\begin{aligned}
\hat{U}_{n}(t, 0) & =\hat{T} \frac{\left(-i \int_{0}^{t} H(\tau) d \tau\right)^{n}}{n !} \\
\hat{U}(t, 0) & =\sum_{n=0}^{\infty} U_{n}(t, 0)=\hat{T} e^{-i \int_{0}^{t} H(\tau) d \tau}
\end{aligned}
$$

The series above is infinite and therefore must be truncated for any practical purpose. Although all the Hamiltonians involved are Hermitian, the truncated series is not (see Appendix A.4 for a full proof) and therefore the truncated propagator is not unitary. A way to circumvent this problem was found by Magnus [76], and is based on the Baker-Campbell-Hausdorff relation. In this approach the time $t$ is divided into small steps:

$$
t=\sum_{k} \tau_{k}
$$

so that the Hamiltonian acting during each step can be considered to be timeindependent. The propagator in Eq. A.6 is therefore the result of an infinite number of rotations in spin space:

$$
\hat{U}(t, 0)=e^{-i H_{n} \tau_{n}} \cdot \ldots \cdot e^{-i H_{2} \tau_{2}} \cdot e^{-i H_{1} \tau_{1}}
$$

which is convenient because, provided all the Hamiltonians involved are Hermitian, the whole propagator is the product of unitary transformations and hence is also unitary. AHT tries to express the entire sequence above by a single transformation under an average Hamiltonian:

$$
\hat{U}(t, 0) \approx e^{-i \bar{H} t}
$$

This is done by invoking the Baker-Campbell-Hausdorff relation:

$$
e^{B} e^{A}=e^{A+B+\frac{1}{2}[B, A]+\frac{1}{12}([B,[B, A]]+[[B, A], A])+\ldots}
$$

Applied to the expansion in Eq. A.8 this gives:

$$
\hat{U}(t, 0) \approx e^{-i \bar{H} t}=e^{-i \sum_{k} H_{k} \tau_{k}-\frac{1}{2} \sum_{k} \sum_{l>k}\left[H_{l} \tau_{l}, H_{k} \tau_{k}\right]+\ldots}
$$

By comparing the second and third terms in Eq. A.11, the form of the average Hamiltonian is readily found:

$$
\bar{H}=\frac{1}{t}\left(\sum_{k} H_{k} \tau_{k}-\frac{i}{2} \sum_{k} \sum_{l>k}\left[H_{l} \tau_{l}, H_{k} \tau_{k}\right]+\ldots\right)
$$

Each term in the expansion represents a different order in the truncation, and these terms are typically referred as the first- and second-order terms of the 
average Hamiltonian. A generalisation of the above equation is obtained by changing the discrete form into an integral. The first two orders in the generalised expansion are:

$$
\begin{aligned}
& \bar{H}^{(1)}=\frac{1}{t} \int_{0}^{t} H\left(t_{1}\right) d t_{1} \\
& \bar{H}^{(2)}=-\frac{i}{2 t} \int_{0}^{t} d t_{2} \int_{0}^{t_{2}}\left[H\left(t_{2}\right), H\left(t_{1}\right)\right] d t_{1}
\end{aligned}
$$

Eq. A.12 can easily be truncated while preserving unitarity. However, it converges at a rate similar to Eq. A.5 and therefore is most useful when only a few terms are needed to describe the problem. Fast convergence is ensured when:

$$
\hat{U}(t, 0) \sim 1 \text { or } H t \ll 1
$$

and a strategy to minimise the number of required terms consists in scaling down the size of the Hamiltonian by expressing it in an interaction frame. This is done following the recipe in Appendix A.3.

\section{A.3. Interaction Frame Transformations}

This appendix derives the equation to be used to express an Hamiltonian in a given interaction frame. It starts from the time-dependent Schrödinger equation:

$$
i \frac{\partial}{\partial t} \Psi=H(t) \Psi
$$

and assumes that the transformation into the interaction frame is performed by an operator $R$ :

$$
\begin{aligned}
& \tilde{\Psi}=R \Psi \\
& \Psi=R^{-1} \tilde{\Psi}
\end{aligned}
$$

Eq. A.16 is inserted into Eq. A.15 to obtain:

$$
\begin{aligned}
i \frac{\partial}{\partial t}\left(R^{-1} \tilde{\Psi}\right) & =H(t) R^{-1} \tilde{\Psi} \\
i\left(\frac{\partial R^{-1}}{\partial t} \tilde{\Psi}+R^{-1} \frac{\partial \tilde{\Psi}}{\partial t}\right) & =H(t) R^{-1} \tilde{\Psi} \\
i R^{-1} \frac{\partial \tilde{\Psi}}{\partial t} & =H(t) R^{-1} \tilde{\Psi}-i \frac{\partial R^{-1}}{\partial t} \tilde{\Psi}
\end{aligned}
$$

Multiplying both terms by $R$ from the left side:

$$
\begin{aligned}
& i \frac{\partial \tilde{\Psi}}{\partial t}=\left(R H(t) R^{-1}-i R \frac{\partial R^{-1}}{\partial t}\right) \tilde{\Psi} \\
& i \frac{\partial \tilde{\Psi}}{\partial t}=\tilde{H} \tilde{\Psi}
\end{aligned}
$$


where

$$
\tilde{H}=\left(R H(t) R^{-1} \tilde{\Psi}-i R \frac{\partial R^{-1}}{\partial t} \tilde{\Psi}\right)
$$

gives a recipe to express a Hamiltonian in any given interaction frame.

\section{A.4. Hermiticity of a combination of Hermitian operators}

This appendix collects some rules on the Hermiticity of combinations of Hermitian operators (all below are assumed to be Hermitian).

1. The expansion in Eq. A.5 is not Hermitian since the product of two Hermitian operators is not Hermitian when the two operators do not commute:

$$
\left(H_{1} H_{2}\right)^{\dagger}=H_{2}^{\dagger} H_{1}^{\dagger}=H_{2} H_{1}
$$

2. The first-order average Hamiltonian term in Eq. A.13 is Hermitian because it is a sum of Hermitian operators.

3. The second-order average Hamiltonian term in Eq. A.13 is also Hermitian because the commutator of two Hermitian operators multiplied by the imaginary unit is Hermitian:

$$
\left(i\left[H_{1}, H_{2}\right]\right)^{\dagger}=\left(i H_{1} H_{2}-i H_{2} H_{1}\right)^{\dagger}=-i\left(H_{2}^{\dagger} H_{1}^{\dagger}-H_{1}^{\dagger} H_{2}^{\dagger}\right)=i\left[H_{1}, H_{2}\right]
$$

Moreover, all even-ordered terms involve $i$ times an odd number of commutators and therefore all even-ordered terms are Hermitian.

4. Third-order (and any odd-order) terms involve an even number of commutators and are therefore also Hermitian:

$$
\begin{aligned}
\left(\left[H_{3},\left[H_{1}, H_{2}\right]\right]\right)^{\dagger} & =\left(H_{3} H_{1} H_{2}-H_{3} H_{2} H_{1}-H_{1} H_{2} H_{3}+H_{2} H_{1} H_{3}\right)^{\dagger} \\
& =\left(H_{2}^{\dagger} H_{1}^{\dagger} H_{3}^{\dagger}-H_{1}^{\dagger} H_{2}^{\dagger} H_{3}^{\dagger}-H_{3}^{\dagger} H_{2}^{\dagger} H_{1}^{\dagger}+H_{3}^{\dagger} H_{1}^{\dagger} H_{2}^{\dagger}\right) \\
& =\left[H_{3},\left[H_{1}, H_{2}\right]\right]
\end{aligned}
$$

\section{A.5. Single-transition spin operators}

Given two generic spin states $|r\rangle$ and $|s\rangle$ it is possible to define the following set of single-transition spin operators [60, 77]:

$$
\begin{aligned}
\mathbf{I}_{x}^{r s} & =\frac{1}{2}(|r\rangle\langle s|+| s\rangle\langle r|) \\
\mathbf{I}_{y}^{r s} & =\frac{1}{2 i}(|r\rangle\langle s|-| s\rangle\langle r|) \\
\mathbf{I}_{z}^{r s} & =\frac{1}{2}(|r\rangle\langle r|-| s\rangle\langle s|) \\
\mathbf{1}^{r s} & =(|r\rangle\langle r|+| s\rangle\langle s|)
\end{aligned}
$$


that satisfies the following commutation rules:

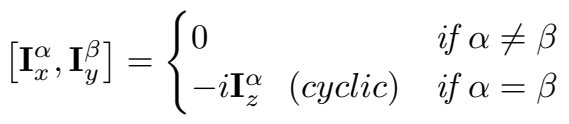

Accordingly, single-transition spin operators for the pair of states $\left|S_{0}\right\rangle$ and $\left|T_{0}\right\rangle$ are defined as:

$$
\begin{aligned}
\mathbf{I}_{x}^{S T} & =\frac{1}{2}\left(\left|S_{0}\right\rangle\left\langle T_{0}|+| T_{0}\right\rangle\left\langle S_{0}\right|\right) \\
\mathbf{I}_{y}^{S T} & =\frac{1}{2 i}\left(\left|S_{0}\right\rangle\left\langle T_{0}|-| T_{0}\right\rangle\left\langle S_{0}\right|\right) \\
\mathbf{I}_{z}^{S T} & =\frac{1}{2}\left(\left|S_{0}\right\rangle\left\langle S_{0}|-| T_{0}\right\rangle\left\langle T_{0}\right|\right) \\
e \mathbf{1}^{S T} & =\left(\left|S_{0}\right\rangle\left\langle S_{0}|+| T_{0}\right\rangle\left\langle T_{0}\right|\right)
\end{aligned}
$$

\section{A.6. Rotation superoperator simplification for J-synchronised echo trains}

In this appendix we demonstrate the following equality (used to simplify Eq. 89):

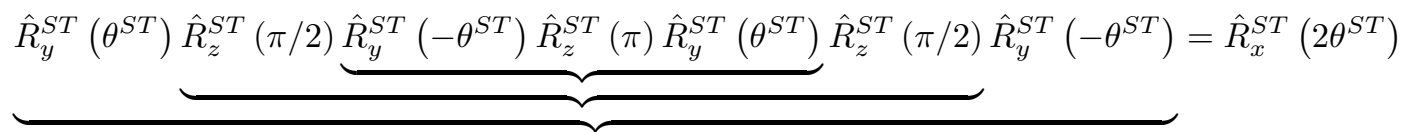

The operator train on the left-hand side of Eq. A.27 can be treated as a series of rotation sandwiches (as indicated by the brackets) that can be solved consecutively from the inner to the outer ones. The inner one is solved by first multiplying from the right-hand side by a unity operator written as $\hat{R}_{z}^{S T}(-\pi) \hat{R}_{z}^{S T}(\pi)$ to give:

$$
\hat{R}_{y}^{S T}\left(-\theta^{S T}\right) \underbrace{\hat{R}_{z}^{S T}(\pi) \hat{R}_{y}^{S T}\left(\theta^{S T}\right) \hat{R}_{z}^{S T}(-\pi)} \hat{R}_{z}^{S T}(\pi)
$$

where the central part is a rotation of $180^{\circ}$ around the z-axis of the $S T$ subspace, resulting in a change of sign of the angle $\theta^{S T}$ in $\hat{R}_{y}^{S T}\left(\theta^{S T}\right)$, thus simplifying the whole expression as:

$$
\hat{R}_{y}^{S T}\left(-\theta^{S T}\right) \underbrace{\hat{R}_{z}^{S T}(\pi) \hat{R}_{y}^{S T}\left(\theta^{S T}\right) \hat{R}_{z}^{S T}(-\pi)} \hat{R}_{z}^{S T}(\pi)=\hat{R}_{y}^{S T}\left(-2 \theta^{S T}\right) \hat{R}_{z}^{S T}(\pi)
$$

This result can be inserted in Eq. A.27, which becomes:

$$
\underbrace{\hat{R}_{y}^{S T}\left(\theta^{S T}\right) \underbrace{\hat{R}_{z}^{S T}(\pi / 2) \hat{R}_{y}^{S T}\left(-2 \theta^{S T}\right) \hat{R}_{z}^{S T}(-\pi / 2)} \hat{R}_{y}^{S T}\left(-\theta^{S T}\right)}
$$


where the inner rotation sandwich is a rotation of $90^{\circ}$ around the z-axis of the $S T$ subspace that gives:

$$
\underbrace{\hat{R}_{z}^{S T}(\pi / 2) \hat{R}_{y}^{S T}\left(-2 \theta^{S T}\right) \hat{R}_{z}^{S T}(-\pi / 2)}=\hat{R}_{x}^{S T}\left(2 \theta^{S T}\right)
$$

This result can be inserted in Eq. A.27, which simplifies to:

$$
\hat{R}_{y}^{S T}\left(\theta^{S T}\right) \hat{R}_{x}^{S T}\left(2 \theta^{S T}\right) \hat{R}_{y}^{S T}\left(-\theta^{S T}\right)
$$

For $\theta^{S T} \ll 1$ Eq. A.32 can be approximated by a rotation of $2 \theta^{S T}$ about the $\mathrm{x}$-axis of the $S T$ subspace. This is readily seen by expanding Eq. A.32 in a Taylor series and retaining the first order terms only:

$$
\begin{aligned}
& \hat{R}_{y}^{S T}\left(\theta^{S T}\right) \hat{R}_{x}^{S T}\left(2 \theta^{S T}\right) \hat{R}_{y}^{S T}\left(-\theta^{S T}\right)=e^{-i \theta^{S T} \mathbf{I}_{y}^{S T}} e^{-i 2 \theta^{S T} \mathbf{I}_{x}^{S T}} e^{i \theta^{S T} \mathbf{I}_{y}^{S T}} \\
& \approx\left(\mathbf{1}^{S T}-i \theta^{S T} \mathbf{I}_{y}^{S T}\right)\left(\mathbf{1}^{S T}-i 2 \theta^{S T} \mathbf{I}_{x}^{S T}\right)\left(\mathbf{1}^{S T}+i \theta^{S T} \mathbf{I}_{y}^{S T}\right)+O\left[\theta^{S T}\right]^{2} \\
& \approx \mathbf{1}^{S T}-i 2 \theta^{S T} \mathbf{I}_{x}^{S T}+O\left[\theta^{S T}\right]^{2} \\
& \approx \hat{R}_{x}^{S T}\left(2 \theta^{S T}\right)
\end{aligned}
$$

\section{Acknowledgement}

I am very grateful to M. H. Levitt and G. Stevanato for useful discussions and careful reading of the manuscript. I would also like to thank current and past colleagues in Levitt's group with whom we devised, discussed, developed and tried out these ideas, in particular, M. C. D. Tayler, P. Håkansson, J. Bocan, J.N. Dumez, J. T. Hill-Cousins, A. I. Pop, L. J. Brown and R. C. D. Brown. Many thanks to EPSRC (EP/I036141/1) and ERC (291044 - HYPERSINGLET) for providing funding for this research.

\section{References}

[1] M. H. Levitt, Singlet and other states with extended lifetimes, in Encyclopedia of Nuclear Magnetic Resonance, R. K. Harris, R. E. Wasylishen, Eds. Wiley, Chichester, UK (2010).

[2] M. H. Levitt, Singlet Nuclear Magnetic Resonance, Ann. Rev. Phys. Chem. 63 (2012) 89-105.

[3] G. Pileio, Relaxation theory of nuclear singlet states in two spin-1/2 systems, Prog. Nucl. Magn. Res. Spectr. 56 (2010) 217-231.

[4] E. Vinogradov, A. K. Grant, Hyperpolarized long-lived states in solution nmr: three-spin case study in low field, J. Magn. Reson. 194 (2008) 46-57.

[5] P. R. Vasos, A. Comment, R. Sarkar, P. Ahuja, S. Jannin, J.-P. Ansermet, J. A. Konter, P. Hautle, B. van den Brandt, G. Bodenhausen, Long-lived states to sustain hyperpolarized magnetization, Proc. Nat. Acad. Sci. 106 (2009) 18469-18473. 
[6] P. Ahuja, R. Sarkar, S. Jannin, P. R. Vasos, G. Bodenhausen, Proton hyperpolarisation preserved in long-lived states, Chem. Comm. 46 (2010) 8192-8194.

[7] A. Bornet, S. Jannin, G. Bodenhausen, Three-field nmr to preserve hyperpolarized proton magnetization as long-lived states in moderate magnetic fields, Chem. Phys. Lett. 512 (2011) 151-154.

[8] M. C. D. Tayler, I. Marco-Rius, M. I. Kettunen, K. M. Brindle, M. H. Levitt, G. Pileio, Direct enhancement of nuclear singlet order by dynamic nuclear polarization, J. Am. Chem. Soc. 134 (2012) 7668-7671.

[9] M. B. Franzoni, L. Buljubasich, H. W. Spiess, K. Münnemann, Longlived ${ }^{1} \mathrm{H}$ singlet spin states originating from para-hydrogen in cs-symmetric molecules stored for minutes in high magnetic fields, J. Am. Chem. Soc. 134 (2012) 10393-10396.

[10] Y. Feng, T. Theis, X. Liang, Q. Wang, P. Zhou, W. S. Warren, Storage of hydrogen spin polarization in long-lived ${ }^{13} \mathrm{C}_{2}$ singlet order and implications for hyperpolarized magnetic resonance imaging, J. Am. Chem. Soc. 135 (2013) 9632-9635.

[11] M. B. Franzoni, D. Graafen, L. Buljubasich, L. M. Schreiber, H. W. Spiess, K. Münnemann, Hyperpolarized ${ }^{1} \mathrm{H}$ long lived states originating from parahydrogen accessed by rf irradiation, Phys. Chem. Chem. Phys. 15 (2013) 17233-17239.

[12] G. Pileio, S. Bowen, C. Laustsen, M. C. D. Tayler, J. T. Hill-Cousins, L. J. Brown, R. C. D. Brown, J.-H. Ardenkjaer-Larsen, M. H. Levitt, Recycling and imaging of nuclear singlet hyperpolarization, J. Am. Chem. Soc. 135 (2013) 5084-5088.

[13] S. Cavadini, J. Dittmer, S. Antonijevic, G. Bodenhausen, Slow diffusion by singlet state nmr spectroscopy, J. Am. Chem. Soc. 127 (2005) 15744-15748.

[14] S. Cavadini, P. R. Vasos, Singlet states open the way to longer time-scales in the measurement of diffusion by nmr spectroscopy, Concepts Magn. Reson. A 32A (2008) 68-78.

[15] R. Sarkar, P. Ahuja, P. R. Vasos, G. Bodenhausen, Measurement of slow diffusion coefficients of molecules with arbitrary scalar couplings via longlived spin states, ChemPhysChem 9 (2008) 2414-2419.

[16] P. Ahuja, R. Sarkar, P. R. Vasos, G. Bodenhausen, Diffusion coefficients of biomolecules using long-lived spin states, J. Am. Chem. Soc. 131 (2009) 7498-7499.

[17] G. Pileio, J.-N. Dumez, I.-A. Pop, J. T. Hill-Cousins, R. C. D. Brown, Realspace imaging of macroscopic diffusion and slow flow by singlet tagging mri, J. Magn. Reson. 252 (2015) 130-134. 
[18] P. Sarkar, P. R. Vasos, G. Bodenhausen, Singlet-state exchange nmr spectroscopy for the study of very slow dynamic processes, J. Am. Chem. Soc. 129 (2007) 328-334.

[19] A. Bornet, P. Ahuja, R. Sarkar, L. Fernandes, S. Hadji, P. R. Vasos, S. Y. Lee, A. Haririnia, D. Fushman, G. Bodenhausen, Long-lived states to monitor protein unfolding by proton nmr, ChemPhysChem 12 (2011) 27292734 .

[20] N. Salvi, R. Buratto, A. Bornet, S. Ulzega, I. Rentero Rebollo, A. Angelini, C. Heinis, G. Bodenhausen, Boosting the sensitivity of ligand-protein screening by nmr of long-lived states, J. Am. Chem. Soc. 134 (2012) 1107611079 .

[21] R. Buratto, D. Mammoli, E. Chiarparin, G. Williams, G. Bodenhausen, Exploring weak ligand-protein interactions by long-lived $\mathrm{nmr}$ states: Improved contrast in fragment-based drug screening, Angew. Chem. 53 (2014) 11376-11380.

[22] R. Buratto, A. Bornet, J. Milani, D. Mammoli, S. Vuichoud, N. Salvi, M. Singh, A. Laguerre, S. Passemard, S. Gerber-Lemaire, S. Jannin, G. Bodenhausen, Drug screening boosted by hyperpolarized long-lived states in nmr., ChemMedChem 9 (2014) 2509-2515.

[23] R. Buratto, D. Mammoli, E. Canet, G. Bodenhausen, Ligand-protein affinity studies using long-lived states of fluorine-19 nuclei, J. Med. Chem. 59 (2016) 1960-1966.

[24] M. Carravetta, M. H. Levitt, Long-lived nuclear spin states in high-field solution nmr, J. Am. Chem. Soc. 126 (2004) 6228-6229.

[25] M. Carravetta, O. G. Johannessen, M. H. Levitt, Beyond the $\mathrm{T}_{1}$ limit: Singlet nuclear spin states in low magnetic field, Phys. Rev. Lett. 92 (2004) 153003.

[26] M. Carravetta, M. H. Levitt, Theory of long-lived nuclear spin states in solution nuclear magnetic resonance. I. Singlet states in low magnetic field, J. Chem. Phys. 122 (2005) 214505.

[27] G. Pileio, M. H. Levitt, Theory of long-lived nuclear spin states in solution nuclear magnetic resonance. II. Singlet spin-locking, J. Chem. Phys. 130 (2009) 214501.

[28] G. Pileio, Singlet state relaxation via intermolecular dipolar coupling, J. Chem. Phys. 134 (2011) 214505.

[29] G. Pileio, Singlet state relaxation via scalar coupling of the second kind, J. Chem. Phys. 135 (2011) 174502. 
[30] M. C. D. Tayler, M. H. Levitt, Paramagnetic relaxation of nuclear singlet states, Phys. Chem. Chem. Phys. 13 (2011) 9128-9130.

[31] E. Vinogradov, A. K. Grant, Long-lived states in solution nmr: Selection rules for intramolecular dipolar relaxation in low magnetic fields, J. Magn. Reson. 188 (2007) 176-182.

[32] G. Pileio, M. H. Levitt, J-stabilization of singlet states in the solution nmr of multiple-spin systems, J. Magn. Reson. 187 (2007) 141-145.

[33] A. K. Grant, E. Vinogradov, Long-lived states in solution nmr: theoretical examples in three- and four-spin systems, J. Magn. Reson. 193 (2008) 177-190.

[34] A. Karabanov, C. Bretschneider, W Köckenberger, Symmetries of the master equation and long-lived states of nuclear spins, J. Chem. Phys. 131 (2009) 204105-10.

[35] G. Pileio, M. Carravetta, M. H. Levitt, Extremely low-frequency spectroscopy of forbidden transitions in nuclear magnetic resonance, Phys. Rev. Lett. 103 (2009) 083002.

[36] W. S. Warren, E. Jenista, R. T. Branca, X. Chen, Increasing hyperpolarized spin lifetimes through true singlet eigenstates, Science 323 (2009) 17111714 .

[37] G. Pileio, M. Carravetta, M. H. Levitt, Storage of nuclear magnetization as long-lived singlet order in low magnetic field, Proc. Nat. Acad. Sci. 107 (2010) 17135-17139.

[38] M. C. D. Tayler, M. H. Levitt, Singlet nuclear magnetic resonance of nearly-equivalent spins, Phys. Chem. Chem. Phys. 13 (2011) 5556-5560.

[39] Y. Feng, R. M. Davis, W. S. Warren, Accessing long-lived nuclear singlet states between chemically equivalent spins without breaking symmetry, $\mathrm{Na}$ ture Phys. 8 (2012) 831-837.

[40] S. J. DeVience, R. L. Walsworth, M. S. Rosen, Preparation of nuclear spin singlet states using spin-lock induced crossing, Phys. Rev. Lett. 111 (2013) 173002 .

[41] T. Theis, Y. Feng, T. Wu, W. S. Warren, Composite and shaped pulses for efficient and robust pumping of disconnected eigenstates in magnetic resonance, J. Chem. Phys. 140 (2014) 014201.

[42] Y. Zhang, P. C. Soon, A. Jerschow, J. W. Canary, Long-lived 1h nuclear spin singlet in dimethyl maleate revealed by addition of thiols, Angew. Chem. 53 (2014) 3396-3399. 
[43] Alexey S. Kiryutin, Herbert Zimmermann, Alexandra V. Yurkovskaya, Hans-Martin Vieth, Konstantin L. Ivanov, Long-lived spin states as a source of contrast in magnetic resonance spectroscopy and imaging, Journal of Magnetic Resonance 261 (2015) $64-72$.

[44] G. Pileio, M. Concistré, M. Carravetta, M. H. Levitt, Long-lived nuclear spin states in the solution nmr of four-spin systems, J. Magn. Reson. 182 (2006) 353-357.

[45] P. Ahuja, R. Sarkar, P. R. Vasos, G. Bodenhausen, Long-lived states in multiple-spin systems, ChemPhysChem 10 (2009) 2217-2220.

[46] B. Meier, J.-N. Dumez, G. Stevanato, J. T. Hill-Cousins, S. Singha Roy, P. Håkansson, S. Mamone, R. C. D. Brown, G. Pileio, M. H. Levitt, Longlived nuclear spin states in methyl groups and quantum-rotor-induced polarization, J. Am. Chem. Soc. 135 (2013) 18746-18749.

[47] A. N. Pravdivtsev, A. V. Yurkovskaya, H. Zimmermann, H. M. Vieth, K. L. Ivanov, Magnetic field dependent long-lived spin states in amino acids and dipeptides, Phys. Chem. Chem. Phys. 16 (2014) 7584-7594.

[48] G. Stevanato, S. Singha Roy, J. T. Hill-Cousins, I. Kuprov, L. J. Brown, R. C. D. Brown, G. Pileio, M. H. Levitt, Long-lived nuclear spin states far from magnetic equivalence, Phys. Chem. Chem. Phys. 17 (2015) 5913-5922.

[49] J.-N. Dumez, P. Håkansson, S. Mamone, B. Meier, G. Stevanato, J. T. Hill-Cousins, S. Singha Roy, Richard C. D. Brown, G. Pileio, M. H. Levitt, Theory of long-lived nuclear spin states in methyl groups and quantumrotor induced polarisation, J. Chem. Phys. 142 (2015) 044506.

[50] M. Emondts, M. P. Ledbetter, S. Pustelny, T. Theis, B. Patton, J. W. Blanchard, M. C. Butler, D. Budker, A. Pines, Long-lived heteronuclear spin-singlet states in liquids at a zero magnetic field, Phys. Rev. Lett. 112 (2014) 077601.

[51] G. Pileio, M. Carravetta, E. Hughes, M. H. Levitt, The long-lived nuclear singlet state of ${ }^{15} \mathrm{~N}$-nitrous oxide in solution, J. Am. Chem. Soc. 130 (2008) $12582-12583$.

[52] R. K. Ghosh, S. J. Kadlecek, J. H. Ardenkjaer-Larsen, B. M. Pullinger, G. Pileio, M. H. Levitt, N. N. Kuzma, R. R. Rizi, Measurements of the persistent singlet state of $\mathrm{N}_{2} \mathrm{O}$ in blood and other solvents-potential as a magnetic tracer, Mag. Reson. Med. 66 (2011) 1177-1180.

[53] G. Pileio, J. T. Hill-Cousins, S. Mitchell, I. Kuprov, L. J. Brown, R. C. D. Brown, M. H. Levitt, Long-lived nuclear singlet order in near-equivalent ${ }^{13}$ C spin pairs, J. Am. Chem. Soc. 134 (2012) 17494-17497. 
[54] G. Stevanato, J. T. Hill-Cousins, P. Håkansson, S. Singha Roy, L. J. Brown, R. C. D. Brown, G. Pileio, M. H. Levitt, A nuclear singlet lifetime of more than one hour in room-temperature solution, Angew. Chem. 54 (2015) 3740-3743.

[55] C. Laustsen, G. Pileio, M. C. D. Tayler, L. J. Brown, R. C. D. Brown, M. H. Levitt, J.-H. Ardenkjaer-Larsen, Hyperpolarized singlet nmr on a small animal imaging system, Mag. Reson. Med. 68 (2012) 1262-1265.

[56] J. N. Dumez, J. T. Hill-Cousins, R. C. D. Brown, G. Pileio, Long-lived localization in magnetic resonance imaging, J. Magn. Reson. 246 (2014) $27-30$.

[57] D. Graafen, M. B. Franzoni, L. M. Schreiber, H. W. Spiess, K. Münnemann, Magnetic resonance imaging of ${ }^{1} \mathrm{H}$ long lived states derived from parahydrogen induced polarization in a clinical system, J. Magn. Reson. 262 (2016) 68-72.

[58] O.W. Sørensen, A universal bound on spin dynamics, J. Magn. Reson. 86 (1990) 435-440.

[59] M. H. Levitt, Unitary evolution, liouville space and local spin thermodynamics, J. Magn. Reson. 99 (1992) 1-17.

[60] R. R. Ernst, G. Bodenhausen, A. Wokaun, Principles of Nuclear Magnetic Resonance in One and Two Dimensions, Oxford University Press, Oxford (1987).

[61] J. Kowalewski, L. Mäler, Nuclear Spin Relaxation in Liquids: Theory, Experiments and Applications, Taylor \& Francis (2006).

[62] A. Bornet, X. Ji, D. Mammoli, S. Vuichoud, J. Milani, G. Bodenhausen, S. Jannin, Long-lived states of magnetically equivalent spins populated by dissolution-dnp and revealed by enzymatic reactions, Chem. A Eur. J. 20 (2014) 17113-17118.

[63] M. C. D. Tayler, I. Marco-Rius, M. I. Kettunen, K. M. Brindle, M. H. Levitt, G. Pileio, Direct enhancement of nuclear singlet order by dynamic nuclear polarization, J. Am. Chem. Soc. 134 (2012) 7668-7671.

[64] D. Mammoli, B. Vuichoud, A. Bornet, J. Milani, J. N. Dumez, S. Jannin, G. Bodenhausen, Hyperpolarized para-ethanol, J. Chem. Phys. B 119 (2015) 4048-4052.

[65] M. H. Levitt et. al., SpinDynamica code for Wolfram Mathematica.

[66] U. Haeberlen, J. S. Waugh, Coherent averaging effects in magnetic resonance, Phys. Rev. 175 (1968) 453-467. 
[67] K. Gopalakrishnan, G. Bodenhausen, Lifetimes of the singlet states under coherent off-resonance irradiation in $\mathrm{nmr}$ spectroscopy, J. Magn. Reson. 182 (2006) 254-259.

[68] S. J. DeVience, R. L. Walsworth, M. S. Rosen, Dependence of nuclear spin singlet lifetimes on rf spin-locking power, J. Magn. Reson. 218 (2012) 5-10.

[69] M. H. Levitt, Spin Dynamics. Basics of Nuclear Magnetic Resonance, Wiley, Chichester (2001).

[70] Alexey S. Kiryutin, Konstantin L. Ivanov, Alexandra V. Yurkovskaya, Hans-Martin Vieth, Nikita N. Lukzen, Manipulating spin hyperpolarization by means of adiabatic switching of a spin-locking rf-field, Phys. Chem. Chem. Phys. 15 (2013) 14248-14255.

[71] Alexey S. Kiryutin, Alexandra V. Yurkovskaya, Nikita N. Lukzen, HansMartin Vieth, Konstantin L. Ivanov, Exploiting adiabatically switched rf-field for manipulating spin hyperpolarization induced by parahydrogen, The Journal of Chemical Physics 143 (2015) 23.

[72] M. V. Berry, Quantal phase factors accompanying adiabatic changes, Proc. R. Soc. Lond. A 392 (1984) 45-47.

[73] J. D. van Beek, M. Carravetta, G. C. Antonioli, M. H. Levitt, Spherical tensor analysis of nuclear magnetic resonance signals, J. Chem. Phys. 122 (2005) 244510.

[74] G. Pileio, M. H. Levitt, Isotropic filtering using polyhedral phase cycles. application to singlet-state nmr, J. Magn. Reson. 191 (2008) 148-155.

[75] M. C. D. Tayler, M. H. Levitt, Accessing long-lived nuclear spin order by isotope-induced symmetry breaking, J. Am. Chem. Soc. 135 (2013) $2120-2123$.

[76] W. Magnus, On the exponential solution of differential equations for a linear operator, Comm. Pure Appl. Math. 7 (1954) 649-673.

[77] S Vega, Fictitious spin-1/2 operator formalism for multiple quantum nmr, J. Chem. Phys. 68 (1978) 5518-5527. 